V. $\mathrm{X}$ AND $\gamma$ RADIATION : STARS AND GALAXIES 


\title{
HIGH ENERGY COSMIC PHOTONS AND NEUTRINOS
}

\author{
by R. J. Gould and G. R. BuRbIDGF
}

(University of California, San Diego, La Jolla, California. U. S. A.)

RÉsumf. - La présente mise au point est consacrée principalement à l'interprétation des observations récentes du rayonnement $X$ et $\gamma$ des sources célestes. On estime les flux probables et on les compare aux observations. On calcule les spectres du rayonnement synchrotron, du bremsstrahlung et de l'effet CoMPTON (inverse) par des électrons relati. vistes. Ces derniers seraient produits (en passant par la création de mésons) par les collisions nucléaires avec des rayons cosmiques, dans le milieu galactique et intergalactique. On calcule aussi les spectres produits par le $\pi^{0}$ decay. On envisage les possibilités d'émissions de rayons $X$ par les étoiles à neutrons, les couronnes stellaires et les restes de supernovae. On considère plus spécialement les processus qui se produisent dans la Nébuleuse du Crabe. En se basant sur des considérations énergétiques, on étudie l'émission de photons de grande énergie par des objets extragalactiques, particulièrement les radio-sources intenses en cours de formation. La possibilité de détecter des neutrinos cosmiques est aussi envisagée.

Pour le moment, aucun processus particulier ne peut être mis en évidence dans aucun des cas où du rayonnement énergétique est observé, bien qu'un certain nombre de conclusions puisse être tiré des résultats d'observation actuellement disponibles. En particulier certaines théories cosmogoniques peuvent déjà étre confrontées avec ces observations.

ABSTRAOT. - This review concentrates primarily on the problem of interpreting the recent $X$-ray and $\gamma$-ray observations of celestial sources. The expected fluxes of hard radiation from various processes are estimated (when possible) and are compared with the observations. We compute the synchrotron, bremsstrahlung, and (inverse) CомтTоN spectra originating from relativistic electrons produced (via meson production) in the galaxy and intergalactic medium by cosmic ray nuclear collisions; the spectra from $\pi^{0}$-decay are also computed. Neutron stars, stellar coronae, and supernova remnants are reviewed as possible $X$-ray sources. Special consideration is given to the processes in the Crab Nebula. Extragalactic objects as discrete sources of energetic photons are considered on the basis of energy requirements; special emphasis is given to the strong radio sources and the possibility of the emission of hard radiation during their formation. The problem of the detection of cosmic neutrinos is reviewed.

As yet, no definite process can be identified with any of the observed fluxes of hard radiation, although a number of relevant conclusions can be drawn on the basis of the available preliminary observational results. In particular, some cosmogonical theories can be tested.

Резюме. - Настоящая обработка посвящена главным образом интерпретации дедавных наблюдений излучений $X$ и $\gamma$ небесных источников. Давы оценки вероятных потоков, которые сравнены с наблюдениями. Вычислены спектры синхротронного излучения, bremsstralhung и эффекта комптона (обратного) для релативистких электронов. Эти последние должно быть произведены (проходя через создание мезонов) ядерными соударениями с космическими лучами, в галактической и межгалактической средах. Вычислены также спектры произведенные распадом. Предвидены возможности эмиссий лучей $\mathrm{X}$ звездами из нейтронов, звездными коронами и остатками сверхновых. Более специально рассмотрены процессы происходящие в туманности Краба. Основываясь на соображедиях энергетического порядка, исследована эмиссия фотонов с больпой энергией внегалактическими обвектами, в частности, интенсивными радиоисточниками в состоянии образования. Предвидена также возможность обнаружить космические нейтрино.

Пока что, никакой особый процесс не может быть выявлен ни в одном из случаев, когда наблюдается энергетическое излучение, хотя некоторое число заключедий может быть выведево из результатов наблюдений имеющихся в настоящее время. В частности, некоторые носмогонические теории могут уже быть сравнены с этими наблюдедиями. 


\section{INTRODUCTION.}

Except for the possible existence of an intergalactic gas, the bulk of the matter in the universe is in the interior of stars. Most of this stellar matter is at a temperature of $\sim 10^{6} \mathrm{oK}$, while in some stages of stellar evolution the temperature may reach $10^{\circ} \mathrm{oK}$. Due to the high opacity of stellar matter the $\mathrm{KeV}-\mathrm{MeV}$ photons emitted in the interior of stars are absorbed and degraded to lower energy before they reach the stellar surface. Thus, the visible stellar matter in the universe lies in the relatively cool photospheres where the temperature is about $10^{3}-10^{5} \mathrm{oK}$ and where the $\sim \mathrm{eV}$ photons observed by optical astronomers are emitted. There are, however, a number of processes in the universe which are capable of producing observable high energy photons ; these processes usually involve high energy non-thermal electrons. Observations of these high energy photons are extremely difficult due to the necessity of carrying out the experiments above the earth's absorbing atmosphere by means of rockets, satellites, or balloons. Nevertheless, during the past few years a number of workers have succeeded in detecting fluxes of energetic cosmic quanta. Although these observations are very preliminary, it is clear that a great deal of knowledge may be gained from the interpretation of this data. Of special interest is the application of the observations to the analysis and testing of cosmological theories (see Sect. $11 \mathrm{~h}, i$ ). We feel that many cosmological questions may be answered in the near future by the analysis of observations of high energy photons and neutrinos. At present this relatively new field of research is in its infancy and our review will probably soon be out of date. It is our hope that it will be of some use as a guide for the interpretation of observations in the near future.

We might summarize briefly some basic physical processes by which energetic photons are produced. First we list the processes which produce continuum radiation : 1) Bremsstrahlung is emitted in the interaction of charged particles with matter. It results from $e-p$ Coulomb scattering at nonrelativistic energies and from both $e-p$ and $e-e$ scatterings at relativistic energies. 2) The Compton scattering of a low energy thermal photon by a high energy electron produces a high energy scattered photon, the energy being transferred from the electron. This process was first discussed by Fenenbera and Prima-
KOFF (1948). FrRMI pointed out that this was probably the mechanism by which electrons were removed from the primary cosmic ray flux. 3) Electrons moving in magnetic fields emit synchrotron radiation; this is the primary mechanism for radio emission in galaxies. Very high electron energies are required to produce high energy photons by this process ; cosmic synchrotron spectra probably extend at most to photon energies in the $\mathrm{keV}-\mathrm{MeV}$ range. 4) Gamma rays result form the decay of $\pi^{0}$-mesons $\left(\pi^{0} \rightarrow 2 \gamma\right)$ following the production of mesons in collisions between primary cosmic ray particles and nuclei of the interstellar and intergalactic gas. Cosmic ray nuclear collisions are also a source of high energy electrons via charged pion production and $(\pi \rightarrow \mu \rightarrow e)$ decay, as was proposed by BURBIDGE and GINZBURG in the early attempts to understand radio sources. A recent discussion applying to galactic radiation has been given by PollaOK and FAzio (1963) and by Ginzburg and Syrovatsky (1964). $\pi^{\circ}$-gammas are also produced following meson production in matter anti-matter annihilation. Some processes which produce line radiation are: 5) Characteristic $\mathrm{X}$-rays are produced following the ejection of an atomic inner shell electron by, for example, a high energy particle or photon flux. The resulting cascade transitions give rise to the emission of $K$, L, etc... - series X-rays. 6) Gamma rays are produced in the annihilation of electrons and positrons $\left(e^{+}+e^{-} \rightarrow 2 \gamma\right)$. Energetic positrons in the interstellar (but not intergalactic) medium come essentially to rest by various energy loss processes (see Sect. II) before annihilating, and the resulting $\gamma$-rays are essentially monoenergetic at about $0.51 \mathrm{MeV}$. 7). The formation of deuterium via $n+p \rightarrow d+\gamma$ (the inverse of photodisintegration) produces a photon of energy $2.23 \mathrm{MeV}$. This is the only low energy nuclear reaction we have listed here which gives rise directly to $\gamma$-radiation. There are many low energy reactions which give rise to $\gamma$-rays either directly or indirectly, but in general they will occur only in stellar interiors so that the $\gamma$-rays do not escape. However, there are some indications that nuclear reactions sometimes take place in stellar surfaces, so that these $\gamma$-rays may be observable. Both the 0.51 and $2.23 \mathrm{MeV}$ lines were mentioned in an early paper by MoRRIsoN (1958) on the subject of gamma ray astronomy.

Most of the basic photon-producing processes are considered in Section II where the background 
spectra from synchrotron radiation, bremsstrahlung, and the Compton process by high energy electrons and from $\pi^{0}$-decay in the interstellar medium, the galactic halo, and the intergalactic medium are computed. The energetic electron spectrum is computed from secondary production via $\pi-\mu$ decay with the pions produced in nuclear collisions (proton-proton) of cosmic rays with the interstellar and intergalactic gas atomic nuclei and from the various electron energy loss processes. In Section III we consider the production (especially of $\mathrm{X}$-rays) by stars, in particular from stellar coronae and from neutron stars. Supernova remnants are treated in Section IV where special consideration is given to the Crab Nebula. The possibility of observing extragalactic discrete sources of energetic quanta is studied briefly in Section V. The problem of detecting cosmic neutrinos is considered in Section VI.

We shall concentrate in this review in attempting to give reasonable theoretical estimates of the fluxes of hard radiation which may be generated in a variety of celestial sources. However, we wish to stress again that our philosophy is that the detection of hard radiation as an observational science is still in a very rudimentary state and the observations to date cannot be used to refine the the theory in any significant way. We reiterate that we can only give plausible estimates of the fluxes to be expected, and some surprises of the kind that have been encountered in radio astronomy over the past fifteen years may well be in store.

\section{Production in the interstellar gas, THE GaLACTIC HaLO, AND THE INTERGALACTIC MEDIUM.}

In this section we consider the general background flux of cosmic photons produced in electromagnetic interactions involving non-thermal particles. A source of high energy particles is provided by the ordinary cosmic rays, in particular the cosmic ray protons, whose energy spectrum is known and extends up to $\sim 10^{20} \mathrm{eV}$. The protons themselves are not efficient at producing photons in direct electromagnetic interactions, due to their large mass. However, high energy electrons can result from nuclear collisions of cosmic rays in which a shower of pions are produced ; the charged pions then decay into electrons via $\pi \rightarrow \mu \rightarrow e$. The energetic " secondary " electrons which result can produce high energy pho- tons by a number of processes, and these will be considered later in this section. The photon spectrum produced by a specific process is determined (among other things) by the electron spectrum, which in turn is determined by the cosmic ray proton spectrum. We shall assume a universal cosmic ray spectrum, that is, except near local sources of cosmic rays, the cosmic ray flux at any place in the universe is assumed to be the same as that measured at the earth. GINZBURG and Syrovatsky (1963) have argued against a universal cosmic ray flux and estimate that the intergalactic cosmic ray density is smaller than the local (galactic) value by a factor $\sim 10^{-3}$. Howver, their reasoning is based on equipartition arguments and is, in our opinion, not convincing. The intergalactic cosmic ray density is just one of a number of poorly known parameters with which one is confronted in making estimates of photon production processes ; for example, the gas density and magnetic field in the intergalactic medium can only be estimated roughly. Of course it may be that there exists a " primary" cosmic ray electron component, where by primary electrons we mean those which may have been accelerated by the same process and in the same sources that produced the cosmic ray protons. This question is open. Recent experiments by $\mathrm{DH}$ Shong, Hildebrand, and Meyer (1964) measuring the electron/positron ratio in the local cosmic ray flux are certainly relevant to this problem but the experiments still do not allow a definite conclusion regarding the primary or secondary origin of these electrons and positrons. We shall consider only the contribution from secondary electrons. It might be remarked that the acceleration of protons without an accompanying acceleration of electrons can be envisaged easily, since the electrons, with their smaller mass, lose energy by electromagnetic processes more readily.

We shall take a universal differential cosmic ray flux given by

$$
d \mathrm{~J}_{p}=\mathbf{K}_{p} \gamma_{p}-\Gamma_{p} d \gamma_{p},
$$

where $d \mathrm{~J}_{p}$ is the number of incident protons per $\mathrm{cm}^{2}$ per second having Lorentz factors $\gamma_{p}\left(=\mathrm{E}_{p} / \mathrm{m}_{p} c^{2}\right)$ within $d \gamma_{p}$ (centered at $\gamma_{p}$ ); here $K_{p}$ and $\Gamma_{p}$ are constants. By appropriate choice of $K_{p}$ and $\Gamma_{p}$ the power law (1) can be used to describe the observed flux for any range of $\gamma_{p}$. The choice $\Gamma_{p}=2.6$, $\mathrm{K}_{p}=100 \mathrm{~cm}^{-2} \mathrm{~s}^{-1}$ fits the observations (cf. SiNGER 1958) over many orders of magnitude of $\gamma_{p}$ in the high energy range. At lower energies the actual flux is smaller than that described by this 
ohoice of $\Gamma_{p}, K_{p}$. The extrapolation from high energies is too large by a factor $\sim 2$ at $\gamma_{p} \sim 100$ and by a factor $\sim 4$ at $\gamma_{p}=10$. Since we are interested in the effects of the high energy cosmic rays we shall adopt the above values for the parameters $\Gamma_{p}, \mathbf{K}_{p}$ in the caloulations outlined in this section. Given the astronomical parameters (gas density, magnetic field, etc...), the cosmic photon fluxes from various processes (synchrotron radiation, bremsstrahlung, Compton effect, etc...) are essentially determined by the cosmic ray spectrum. However, due to uncertainties in our knowledge of the physics of certain processes, in particular that of meson production in high energy nuclear collisions, the calculated photon fluxes must be considered at best only order of magnitude estimates. Uncertainties in the astronomical parameters further complicate the interpretation of the results. In view of this, a number of simplifying assumptions and approximations are made in the calculation of the physical processes.

After discussing meson production in cosmic ray collisions (part a) the electron production spectrum is derived in (b). Electron energy losses in the galaxy and intergalactic medium are treated in $(c)$ and $(d)$ and the resulting electron spectra are derived in (e). The photon fluxes are calculated in $(f)$ and a discussion and comparison with the observational results follows. Some cosmological considerations of photon production in the intergalactic medium are given in $(h),(i)$.

\section{a) Meson Production in Cosmic Ray Nuclear Collisions.}

All of the laboratory results on meson production are for incident proton energies less than $10 \mathrm{BeV}$ at which it is possible energetically to produce only a few relatively low energy pions per inelastic collision. Our knowledge of meson production by high energy protons is based primarily on theory, and the theories of meson production are very crude ; of course, an accurate theoretical treatment of the problem would be extremely difficult, probably beyond our present knowledge of elementary particle interactions. The simplest theory of meson production in high energy nuclear collisions is that of FrRMI (cf. MARSHAK 1952) and is outlined briefly below, (i). The theory predicts the correct shape for the spectrum of high energy $\gamma$-rays resulting from $\pi^{0^{\prime} s}$ produced in cosmic ray collisions (ii).

(i) Fermi Theory of Meson Production.

Consider the collison of a proton of (lab) energy $\gamma_{p} m_{p} c^{2}$ incident on a proton at rest. In the center of mass (c.m.) system the total energy of the two protons is $2 \bar{\gamma}_{p} m_{p} c^{2}=\left[2\left(\gamma_{p}+1\right)\right]^{\frac{1}{2}} m_{D} c^{2}$, where $\bar{\gamma}_{p}$ is the Lorentz factor of the protons in the c.m. system. Each proton carries a cloud of virtual pions ; in the proton's rest frame the radius of this cloud is approximately $\Lambda_{\pi}=\hbar / m_{\pi} c$, where $m_{\pi}$ is the pion mass. The interaction cross section is then $\sigma \sim \pi \Lambda_{\pi}^{2}$. In the c.m. system each cloud is contracted in the direction of motion by a factor $\bar{\gamma}_{p}$, and when the protons collide, the maximum common volume of the meson clouds (which, presumably, is when the interaction is strongest) is

$$
\Delta \mathrm{V}=\frac{4 \pi}{3} \Lambda_{\pi}^{2} \frac{1}{\gamma}
$$

For high proton energies it is possible energetically to produce many pions in an inelastic collision and Ferm made the assumption that the interaction in the volume (2) was strong enough to produce a distribution of pion energies corresponding to thermal equilibrium with most of the initial proton kinetic energy having been fed into the pion gas. Also, the pions are predominantly highly relativistic and thus have a Planckian distribution. The "temperature" for this distribution is easily shown to be $k \mathrm{~T} \approx \gamma_{\nu}^{\frac{1}{4}} m_{\pi} c^{2}$, so that in the c.m. system the mean pion energy corresponds to

$$
<\bar{\gamma}_{\pi}>\approx \gamma_{p}{ }^{\frac{1}{4}},
$$

and in the lab system (where one of the protons is initially at rest)

$$
\left\langle\gamma_{\pi}\right\rangle \approx \dot{\gamma}_{p} \gamma_{D}{ }^{i} \approx \gamma_{p}^{3 / 4} .
$$

FERMI assumed that the distribution arising when the pion clouds of the colliding protons overlap is " frozen in ", so that equation (4) would apply to the pions produced in the collision. Equation (4) also implies that the multiplicity of pions produced is proportional to (and is, in fact, roughly given by) $\gamma_{p}^{\frac{1}{4}}$.

$A$ number of attempts have been made to improve the Fram theory and some authors have taken a quite different approach to the problem. However, these alternative theories usually predict a pion production spectrum not radically different from that of the Ferm theory. The assumption of thermal equilibrium in the FrRMI theory has been questioned by LaNDaU (1953), who has developed his own theory of meson production. Another defect in the simple FrRMI 
theory is that the effects of the production of other unstable particles (for example K-mesons), which eventually decay into pions, has not been taken into account. Nevertheless, for our purposes essentially the only result which need be specified is the relation between multiplicity (and mean pion energy) and $\gamma_{p}$. The detailed shape of the pion energy spectrum produced by an incident proton of given energy need not concern us.

\section{(ii) Pion Production Spectrum.}

The number of pions produced per second per $\mathrm{cm}^{3}$ within the energy range $d \gamma_{\pi}$ in $p-p$ collisions would be computed from

$$
q_{\pi}\left(\gamma_{\pi}\right) d \gamma_{\pi}=\int d J_{p} n_{\mathrm{H}} \sigma f\left(\gamma_{\pi} ; \gamma_{p}\right) d \gamma_{\pi}
$$

where $d J_{p}$ is the differential incident cosmic ray proton flux, $n_{\mathrm{H}}$ the local density of hydrogen nuclei, $\sigma\left(\approx \pi \Lambda_{\pi}^{2}\right)$ the total (excluding the multiplicity factor) cross section for the event, and $f\left(\gamma_{\pi} ; \gamma_{p}\right)$ the distribution function for the pion production spectrum. We approximate the spectrum $f\left(\gamma_{\pi} ; \gamma_{p}\right)$ by a product of the multiplicity $\left(\approx \gamma_{p_{D}}^{1 / 4}\right)$ and a $\delta$-function at the mean energy $\left(\approx \gamma_{p}^{3 / 4}\right)$ of the pion spectrum for given $\gamma_{p}$ :

$$
f\left(\gamma_{\pi} ; \gamma_{p}\right) \approx \gamma_{p}{ }^{1} \delta\left(\gamma_{\pi}-\gamma_{p}{ }^{3 / 4}\right) \text {. }
$$

With a cosmic ray spectrum given by the power law (1) we then obtain

$$
\begin{aligned}
q_{\pi}\left(\gamma_{\pi}\right) & \approx(4 \pi / 3) \Lambda_{\pi}^{2} \mathrm{~K}_{p} n_{\mathrm{H}} \gamma_{\pi}^{-\Gamma \pi}, \\
\Gamma_{\pi} & =\frac{4}{3}\left(\Gamma_{p}-\frac{1}{2}\right) .
\end{aligned}
$$

The $\delta$-function approximation (6) does not introduce appreciable error. For example, if one computes $q_{\pi}\left(\gamma_{\pi} ; \gamma_{p}\right)$, using the Wien approximation to the Planck thermal distribution, one obtains a slowly varying function of $\gamma_{\pi}$ times $\gamma_{\pi}$ to the power $-\frac{4}{3}\left(\Gamma_{p}-\frac{1}{2}\right)$, that is, essentially the same result as equation (7). Moreover, the exponent in the spectrum (7) will be the same for the case where the mass of the incident cosmic ray particle is different from that of the " target" nucleus. In such a case the analysis follows analogously, since the Lorentz factors in the c.m. system are still proportional to $\gamma^{\frac{1}{2}}$ (when $\gamma$ is large), where $\gamma$ is the Lorentz factor of the incident particle in the rest frame of the target particle. (iii) An Experimental Test for $q_{\pi}\left(\gamma_{\pi}\right)$.

For nuclear collisions at high energy the number of $\pi^{+}, \pi$, and $\pi^{0}$ mesons produced are the same, as is their energy distribution. The $\pi^{0}$ decays via $\pi^{0} \rightarrow 2 \gamma$, with the mean (lab) $\gamma$-ray energy being roughly $\mathrm{E}_{\pi} / 2$. Thus, a measurement of the $\gamma$-ray spectrum from $\pi^{0}$-mesons produced in primary cosmic ray events would give the pion source spectrum $q_{\pi}\left(\gamma_{\pi}\right)$. Recently, KIDD (1963) has measured the spectrum of high energy $\gamma^{\prime} s$ from $\pi^{0}$-mesons produced by cosmic rays at the top of the atmosphere. By performing the experiment at high altitudes he was able to observe $\gamma^{\prime} s$ from $\pi^{\circ} s$ produced predominantly in primary jets. KIDD found for the differential energy spectrum of the $\gamma$-ray flux a power law with exponent $\Gamma_{0}=2.9_{-0.2}^{+0.3}$. The $\gamma$-ray energy range observed by $\mathrm{KmD}$ was

$$
0.7 \times 10^{11} \mathrm{eV}<\mathrm{E}_{0}<10^{12} \mathrm{eV},
$$

corresponding to

$$
10^{3}<\gamma_{\pi}<10^{4} \text { and } 10^{4}<\gamma_{p}<2 \times 10^{5} .
$$

At these proton energies the cosmic ray spectrum is described by the high energy fit with $\Gamma_{p}=2.6$. The corresponding $\Gamma_{\pi}$ from equation (7) is 2.8 and is consistant with the value $\left(\Gamma_{0}\right)$ measured by KIDD. We should like to emphasize that KIDD's experiment confirms the results of the Fermi theory, but not the fundamentals of the theory itself.

\section{b) The Electron Production Spectrum.}

In the charged pion decay $\left(\pi^{ \pm} \rightarrow \mu^{ \pm}+\nu\right)$ most of the center of mass kinetic energy released to the products $\mu, \nu$ is carried away by the neutrino whose energy is small compared with $m_{\pi} c^{2}$. The resulting lab energy of the muon is then approximately $\left(m_{\mu} / m_{\pi}\right) \mathbf{E}_{\pi}$, where $\mathbf{E}_{\pi}$ is the lab energy of the pion before decay. The electron resulting from the muon decay $\left(\mu^{ \pm} \rightarrow e^{ \pm}+2 v\right)$ is highly relativistic and behaves kinematically like the two neutrinos in the decay products. Thus, the mean energy in the spectrum of electron energies is about $\frac{1}{3} m_{\mu} c^{2}$ in the rest frame of the $\mu$, and the mean lab energy $<\mathbf{E}_{e}>$ of the electron resulting from the $\pi \rightarrow \mu \rightarrow e$ decay is roughly

thus,

$$
\frac{1}{3}\left(m_{\mu} / m_{\pi}\right) \mathrm{E}_{\pi} \approx \frac{1}{4} \mathrm{E}_{\pi}
$$

$$
\left\langle\gamma_{e}>\approx \frac{1}{4}\left(m_{\pi} / m_{e}\right)<\gamma_{\pi}>.\right.
$$


Approximating the electron spectrum $f\left(\gamma_{e} ; \gamma_{\pi}\right)$ by a $\delta$-function at this energy we get, for the electron source spectrum,

(8) $q_{e}\left(\gamma_{e}\right) d \gamma_{e} \approx \frac{2}{3} \int q_{\pi}\left(\gamma_{\pi}\right) d \gamma_{\pi} \delta\left(\gamma_{e}-\frac{m_{\pi}}{4 m_{e}} \gamma_{\pi}\right) d \gamma_{e}$ $=\frac{8 m_{e}}{3 m_{\pi}} q_{\pi}\left(\frac{4 m_{e}}{m_{\pi}} \gamma_{e}\right) d \gamma_{e}$;

a factor $\frac{2}{3}$ has been introduced because only charged pions decay into electrons.

We shall consider production and energy losses of electrons with $10^{2} \leqslant \gamma_{\theta} \leqslant 10^{10}$ corresponding to $1 \leqslant \gamma_{\pi} \leqslant 10^{8}$ and to $1 \leqslant \gamma_{\nu} \leqslant 10^{11}$.

\section{c) Electron Energy Losses in the Galaxy.}

Here we consider the various processes tending to decrease the energy of high energy electrons in the galaxy. We calculate the average rate of energy loss in the galaxy which we consider as the region within the galactic halo of radius $\mathbf{R}_{h} \sim 5 \times 10^{22} \mathrm{~cm}$. Actually, energy losses involving interactions with the galactic gas occur predominantly near the plane of the galaxy where most of the gas lies and where the gas is predominantly unionized. The volume of this disk of galactic interstellar gas is $\sim 10^{-2}$ of the volume of the galactic halo.

\section{(i) Ionization Losses.}

The energy loss due to ionization and excitation of the interstellar gas may be computed from Bethe's formula for the stopping power. For high energy electrons this formula is

(9) $\quad-\left(\frac{d \mathrm{E}_{e}}{d t}\right)_{\mathrm{I}}=\frac{2 \pi n e^{4}}{m_{e} c^{2}} \ln \frac{\gamma_{e}^{8} m_{e}^{2} c^{4}}{2 \mathrm{I}_{0}^{2}}$,

where $I_{0}$ is the mean excitation energy of the stopping material (hydrogen), and $n$ is the number density of atoms of the material. The argument of the logarithm in equation (9) is very large and $I_{0}$ may be set equal to the Rydberg energy $\frac{1}{2} \alpha^{2} m_{e} c^{2}\left(\alpha^{-1} \approx 137\right)$. We then have for the ionization loss in a hydrogen gas of mean density $\langle n\rangle$ :

$$
\text { (10) }-<d \gamma_{e} d t>_{\mathrm{I}}=2 \pi c r_{0}^{2}<n>\ln \left(2 \gamma_{e}^{8} / \alpha^{4}\right)
$$

Here $r_{0}\left(=e^{2} / m_{e} c^{2}\right)$ is the classical electron radius. The energy loss computed from equation (10) is shown as a function of $\gamma_{e}$ in Figure 1

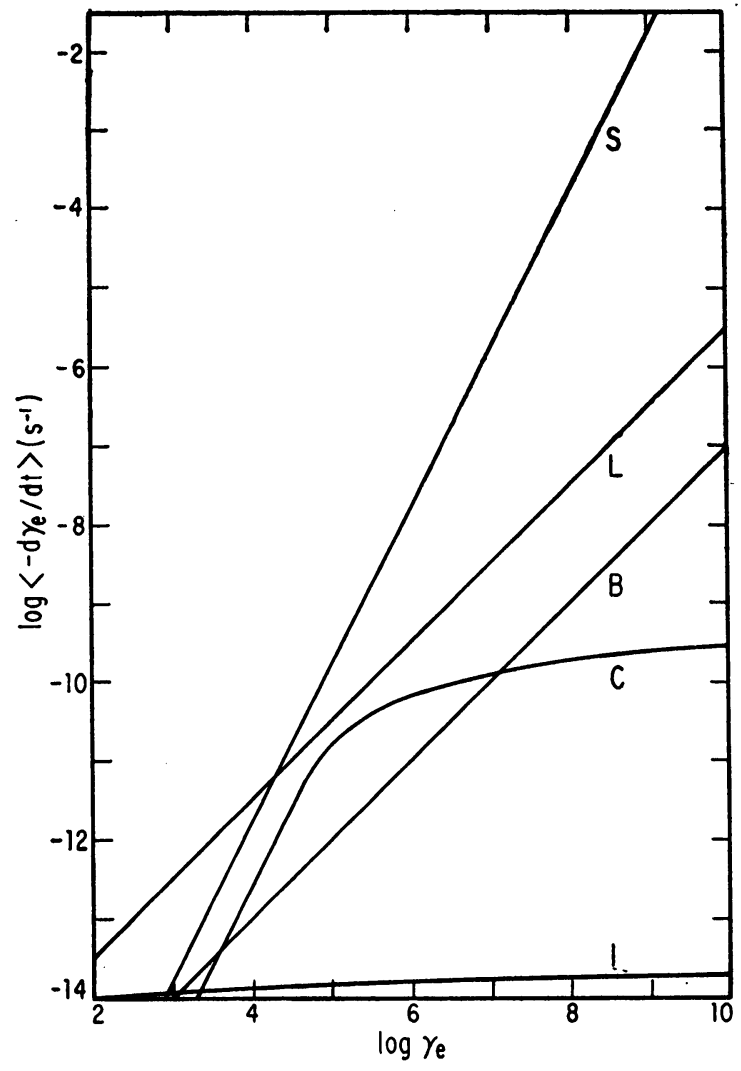

Fig. 1. - Electron energy loss rate in the Galaxy by synchrotron emission (S), leakage out of the halo (L), bremss. trahlung (B), Compton soattering (C), and ionization (I).

for a mean gas density $\langle n\rangle=0.03 \mathrm{~cm}^{-3}$. This mean galactic gas density corresponds to a mean density near the plane of the galaxy of $3 \mathrm{~cm}^{-3}$. This value $\left(3 \mathrm{~cm}^{-3}\right)$ is about three times the observed density of atomic hydrogen. The higher value may be more appropriate if there is a high abundance of interstellar molecular hydrogen (GOULD, GOLD and SALPETER 1963)

\section{(ii) Bremsstrahlung.}

The energy loss rate by bremsstrahlung emission would be computed from

$$
-(d \mathrm{E} / d t)_{\mathrm{B}}=n c \int^{\circ} \hbar \omega d \sigma_{\mathrm{B}}^{\prime},
$$

where $n$ is the density of hydrogen nuclei and $d \sigma_{\mathbf{B}}$ is the differential cross section for the emission of a bremsstrahlung photon of energy within $\hbar d \omega$; in equation (11) the integral is over $\omega$ from 0 to $\gamma_{e} m_{e} c^{2} / \hbar$. For $d \sigma_{\mathrm{B}}$ we take the approximate simplified expression (see JAUCH and ROHRLIOH 1955)

$$
d \sigma_{\mathrm{B}} \approx 4 \alpha r_{0}^{2} \omega^{-1} d \omega \ln 2 \gamma_{\odot}
$$


and calculate the mean bremsstrahlung loss rate:

$$
\text { (12) }-<d \gamma_{e} / d t>_{\mathrm{B}} \approx 4 c \alpha r_{0}^{2}<n>\gamma_{e} \ln \gamma_{e} \text {. }
$$

This is the bremsstrahlung loss rate for interaction of electrons with protons and would be appropriate for calculating the energy loss in regions of ionized hydrogen. Actually, most of the galactic bremsstrahlung is likely to be produced near the galactic plane where the gas is predominantly atomic or molecular, and a correction for the associated shielding effects of the atomic electrons must be made. In fact, for the electron energies of interest the strong shielding expression would be more appropriate. In this case the argument of the logarithm in equation (12) should be replaced by $\sim 137$ (see HeITLer 1954). Using this corrected expression the bremsstrahlung loss rate was computed for $\langle n\rangle=0.03 \mathrm{~cm}^{-3}$ and is shown in Figure 1.

(iii) Synchrotron Losses.

It is well known that a highly relativistic electron of energy $\mathrm{E}_{e}$ in a magnetic field $\mathrm{H}$ moves in a circle with a Larmor radius $r_{\mathrm{L}}=\mathrm{E}_{e} / e \mathrm{H}$ and radiates energy by the synchrotron process at a rate

$$
-<d \mathrm{E}_{e} / d t>_{\mathrm{s}}=\frac{2}{3} c r_{0}^{2}<\mathrm{H}^{2}>\gamma_{e}^{2}
$$

The frequency spectrum of the radiation consists in a continuum with a maximum around $\nu_{\mathrm{L}} \gamma_{e,}^{2}$, $\nu_{L}\left(=e H / 2 \pi m_{e} c\right)$ being the Larmor frequency. The loss rate $-<d \gamma_{e} / d t>_{\mathrm{s}}$ is shown in Figure 1 for a magnetic field $\mathrm{H}=3 \times 10^{-6}$ gauss corresponding to the galactic halo.

\section{(iv) Compton Scattering by Stellar Photons.}

The Compton process, whereby a high energy electron makes an elastic collision with a thermal stellar photon, and transfers some of its kinetic energy to the photon, has been considered in some detail by Femenberg and Primakoff (1948) and by Donarue (1951). More recently, Felten and MORRISON (1963) have suggested this process as a mechanism for producing energetic photons. Consider the collision between an electron of energy $\gamma_{e} m_{e} c^{2}$ and a thermal photon of the galactic radiation field of initial energy $\varepsilon_{r}$. Let $\varepsilon_{r}$ denote the photon energy after scattering; let $\varepsilon_{r}^{*}$ denote the initial energy of the photon in the rest frame of the electron $; \varepsilon_{r}^{*} \approx \gamma_{e} \varepsilon_{r}$. For $\varepsilon_{r}^{*} \ll m_{e} c^{2}$ the cross section for the scattering process is given by the THомpson limit :

$$
\sigma_{\mathrm{I}} \rightarrow \frac{8 \pi}{3} r_{0}^{2}
$$

while the mean energy loss per scattering may easily be shown, by the kinematics of the problem, to be

$$
\left(\bar{\varepsilon}_{r}^{\prime}\right)_{\mathrm{I}} \approx \gamma_{e}^{2} \varepsilon_{r} .
$$

For collisions with very high energy electrons in which $\varepsilon_{r}^{*} \gg m_{e} c^{2}$, the KLEnN-NISHTNA formula must be used to compute the scattering cross section. For high energies this formula approaches

$$
\sigma_{\mathrm{II}} \rightarrow \pi r_{0}^{2} \frac{m_{e} c^{2}}{\varepsilon_{r}^{*}} \ln \frac{2 \varepsilon_{r}^{*}}{m_{e} c^{2}},
$$

while the mean energy loss per scattering is now comparable to the initial energy of the electron :

$$
\left(\bar{\varepsilon}_{r}^{\prime}\right)_{\text {II }} \approx \gamma_{e} m_{e} c^{2} \text {. }
$$

The electron energy loss is computed from

$$
\text { (18) }-<d \mathrm{E}_{e} / d t>_{\mathrm{c}}=c<\int \sigma n_{r}\left(\varepsilon_{r}\right) \bar{\varepsilon}_{r}^{\prime} d \varepsilon_{r}>\text {, }
$$

where $n_{r}\left(\varepsilon_{r}\right) d \varepsilon_{r}$ is the number density of photons of energy within $d \varepsilon_{r}$ in the radiation field. We shall lump the stellar radiation field into one mean photon energy $\bar{\varepsilon}_{r \text {. }}$ Then

$$
\int n_{r}\left(\varepsilon_{r}\right) d \varepsilon_{r} \equiv n_{r} \rightarrow \rho_{r} / \varepsilon_{r}
$$

where $p_{r}$ is the radiation energy density and $n_{r}$ the number density of photons. For a thermal (black body) radiation field $\bar{\varepsilon}_{r}$ is approximately $3 k \mathrm{~T}_{0}$, where $\mathrm{T}_{0}$ is the temperature of the thermal distribution. By employing the expressions for $\sigma$ and $\bar{\varepsilon}_{r}^{\prime}$ for the low energy region (I) where $\gamma_{e} \ll m_{e} c^{2} / \bar{\varepsilon}_{r}$ and the high energy region (II) where $\gamma_{e} \gg m_{e} c^{2} / \bar{\varepsilon}_{r}$ we get for the energy loss rates :

(19 I)

$$
\begin{aligned}
& -<\frac{d \gamma_{e}}{d t}>_{\mathrm{CI}}=\frac{8 \pi}{3} \frac{r_{0}^{2}}{m_{e} c}<\rho_{r}>\gamma_{e}^{2}, \\
& -<\frac{d \gamma_{e}}{d t}>_{\mathrm{CII}}=\pi r_{0}^{2} m_{e} c^{3} \leq \frac{\rho_{r}>}{\bar{\varepsilon}_{r}^{2}} \ln \frac{2 \gamma_{e} \varepsilon_{r}}{m_{e} c^{2}} .
\end{aligned}
$$

It is interesting to note that at low energies the energy loss rate is proportional to the radiation energy density $\left\langle p_{r}\right\rangle$ while at high energies it is essentially proportional to $\left\langle n_{r}\right\rangle \bar{\varepsilon}_{r}$. Most 
of the contribution to the radiation field in the galactic halo comes from the relatively cool stars in the nuclear region of the galaxy. We shall take $\bar{\varepsilon}_{r}=3 \mathrm{eV}$ and $<\rho_{r}>=10^{-13} \mathrm{erg} / \mathrm{cm}^{3}$ as representative values for the radiation field in the halo. The corresponding energy loss rate is shown in Figure 1. The curves for regions I and II were joined smoothly.

\section{(v) Leakage Out of the Galactic Halo.}

Even for electron energies as high as $\gamma_{e} \sim 10^{10}$ the Larmor radii are only $\sim 1 \mathrm{pc}$, which, presumably is much less than the scale of " magnetic field condensations" in the halo. For this reason the high energy electrons moving in the halo are likely to penetrate only the outer edges of the magnetic field regions, and the paths of the electrons would resemble that of Brownian motion. The mean free path would correspond to motion between magnetic field condensations and, because of the smallness of the electrons' Larmor radii, would be independent of energy if the magnetic field between the condensations were very small. The mean leakage time $\tau_{\mathrm{L}}$ for escape from the halo would be roughly

$$
\tau_{\mathbf{L}} \sim \mathbf{R}_{h}^{2} / \lambda c
$$

where $R_{h}\left(\approx 5 \times 10^{22} \mathrm{~cm}\right)$ is the radius of the halo and $\lambda$ is the mean free path for Brownian motion. The appropriate value of $\lambda$ to be used to calculate $\tau_{\mathrm{L}}$ is very uncertain. In the galactic disk the mean distance between gas clouds is $\sim 100 \mathrm{po} ; \lambda$ for the halo is probably larger than this. Taking $\lambda=1 \mathrm{kpc}$ we calculate $\tau_{\mathrm{L}} \sim 3 \times 10^{15} \mathrm{~s}$.

In a leakage process the energy of the electron is not lost gradually ; instead essentially the total energy of the particle is lost (to the intergalactic medium) instantaneously. The equivalent loss rate is then

$$
-<d \gamma_{e} / d t>_{\mathrm{L}}=\gamma_{e} / \tau_{\mathrm{L}}
$$

and this quantity is plotted in Figure 1 for $\tau_{\mathrm{L}}=3 \times 10^{15} \mathrm{~s}$.

\section{d) Electron Production and Energy Losses in the Intergalactic Medium.}

The calculation of processes in the intergalactic medium is made difficult by our lack of knowledge of the astronomical parameters such as the gas density and magnetic field. Here we shall present results for assumed values of the para- meters. The calculated production rates and energy losses are simply related to the parameters and can be easily revised when better astronomical data are available. Actually, it may be that some additional knowledge of these poorly known data may be gained from further interpretation of the high energy cosmic photon experiments.

As mentioned earlier, we assume a universal cosmic ray flux. The pion and electron production rates are then proportional to the intergalactic gas density and this gas is very likely to be predominantly hydrogen. Observationally, the upper limit to the intergalactic density of atomic hydrogen is $\sim 10^{-5} \mathrm{~cm}^{-3}$ (Freld 1962 ; Davis 1964); the amount of ionized hydrogen is unknown. The usually assumed total density of intergalactic hydrogen is $\left\langle n_{\mathrm{H}}\right\rangle \sim 10^{-5} \mathrm{~cm}^{-3}$; this is the so-called cosmological ( $\left.{ }^{1}\right)$ value and is the figure which we shall adopt. Also, we shall assume that the intergalactic hydrogen is fully ionized. We adopt $10^{-7}$ gauss for the mean intergalactic magnetic field. Certainly the intergalactic modium must have some, if only random, magnetic field. The intergalactic radiation field can be estimated with some reliability. The contribution from all galaxies in the universe resuits in a radiation field similar to the galactic (halo) field but diluted by about a factor of ten. Thus we take $\left\langle p_{r}\right\rangle=10^{-14} \mathrm{erg} / \mathrm{cm}^{3}$ and, again $\bar{\varepsilon}_{r}=3 \mathrm{eV}$.

Assuming the above values for the gas density, magnetic field, and radiation density in the intergalactic medium the various processes can be calculated readily by employing the relations given in part (c) of this section for galactic processes. However, for the bremsstrahlung contribution one must include the effects of electronelectron bremsstrahlung $\mathbf{B}_{e e}$ (see $\mathrm{J}_{\mathrm{AUCH}}$ and ROHRLICH 1955) as well as the contribution from $B_{e p}$. Since the cross section for high energy $B_{e e}$ is approximately equal to that for $B_{e p}$, and since $n_{e}=n_{p}$ for the assumed fully ionized intergalactic medium, the total bremsstrahlung loss $-\left\langle d \gamma_{e} / d t\right\rangle_{\mathrm{B}}$ is given by simply twice the

(1) Several cosmological theories including HoYLE's formulation of the steady-state theory, lead to values of this order for the mean density in the universe. One can arrive at this result by simply setting $E_{0}+V=0$, where $E_{0}$ ( $=\mathrm{Mc}^{2} ; \mathrm{M}$ is the " mass of the universe") is the rest energy of the universe, and $V\left(\sim-G^{2} / R ; R\right.$ is the " radius of the inverse" or HUBBLE radius) is the gravitational energy. The resulting mean density is about two orders of magnitude greater than the observed smeared out density $(\sim 3 \times 10-81$ $\mathrm{gm} / \mathrm{cm}^{3}$ ) from galaxies. The bulk of the matter in the universe is then attributed to the uncondensed intergalactic gas. 
expression (12) with $\langle n\rangle=10^{-5} \mathrm{~cm}^{-3}\left({ }^{1}\right)$.

The " ionization losses" for the fully ionized intergalactic medium actually correspond to production of plasma oscillations. The associated expression for the electron energy loss at high energies reduces to (see HaYakawa and KITAO, 1956)

$$
-<\frac{d \gamma_{e}}{d t}>_{\mathrm{P}}=4 \pi r_{0}^{2} c<n>\ln \frac{2 m_{e} c^{2} \gamma_{e}}{\hbar \omega_{\nu}}
$$

where $\omega_{p}\left(=\left[4 \pi e^{2}<n>/ m_{e}\right]^{1 / 2}\right)$ is the plasma frequency. The result is plotted in Figure 2.

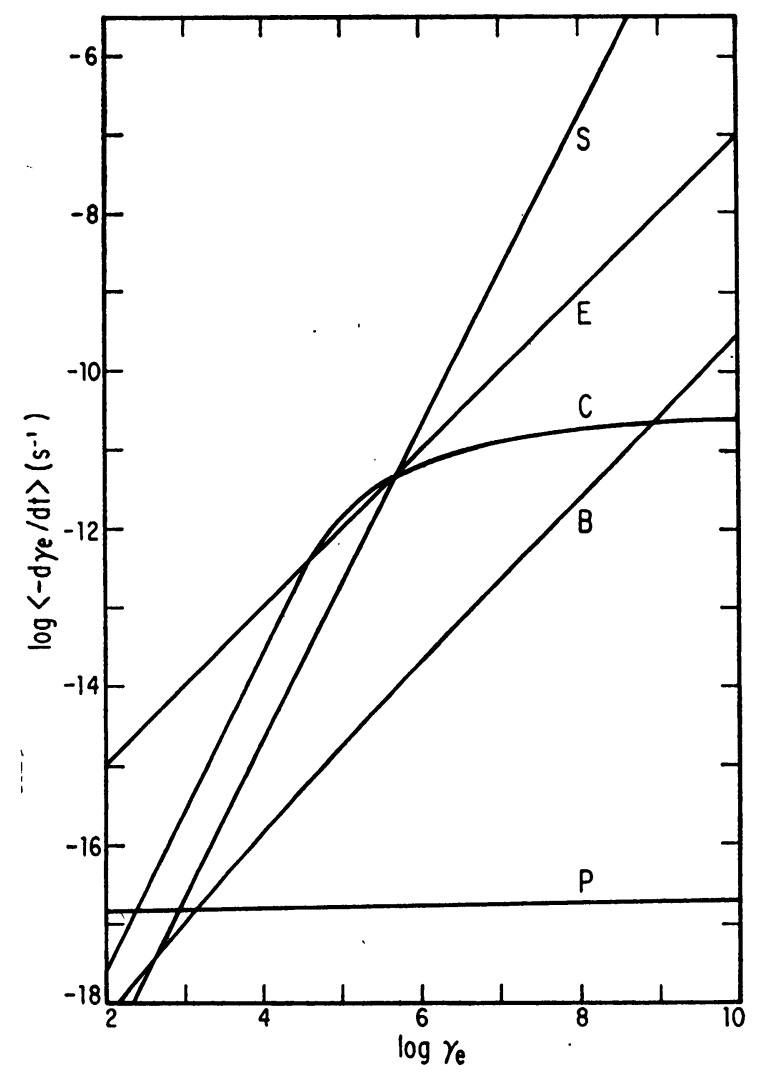

Fra. 2. - Electron energy loss rate in the intergalactic medium by synchrotron emission (S), cosmic expansion (E), Compton scattering (C), bremsstrahlung (B), and excitation of plasma oscillations $(P)$.

For the intergalactic medium one should consider another " effective" energy loss process. The expansion of the universe results in an effective energy loss for the electrons in a given volume of

$$
-<d \gamma_{e} / d t>\stackrel{\cdot}{=} \gamma_{e} / \tau_{\mathrm{E}}
$$

(1) Although $B_{e e} \ldots B_{e p}$ for highly relativistic electrons, for non-relativistic electrons $\mathbf{B}_{e e} \ll \mathbf{B}_{e p}$. Essentially, this is because the photon emission by the non-relativistic system results from the dipole moment formed by the e-p system. where $\tau_{E}$ is the characteristic expansion time given by $\frac{1}{3} \mathrm{H} \sim 10^{17}$ s. $\left(\mathrm{H}^{-1}=\right.$ Hubble constant $)$ The factor $\frac{1}{3}$ takes into account the fact that the expansion is three dimensional, that is, $\mathrm{H}^{-1}$ is the characteristic time for the one dimensional expansion. The effective energy loss due to expansion is plotted in Figure 2 for $\tau_{\mathrm{E}}=10^{17} \mathrm{~s}$, along with the energy losses due to bremsstrahlung, synchrotron radiation, and Compton scattering.

\section{e) The Electron Energy Spectrum in the Halo and Intergalactic Medium.}

Here we consider the electron spectrum which results from production (via $\pi-\mu-e$ decay) in nuclear collisons of cosmic rays and from the various loss processes. Let $n_{e}\left(\gamma_{e}\right) d \gamma_{e}$ denote the number of electrons per $\mathrm{cm}^{3}$ with energies within $m_{e} c^{2} d \gamma_{e}$. The spectral electron density $n_{e}\left(\gamma_{e}\right)$ satisfies a continuity equation in $\gamma_{e}$ (energy space :

$$
\frac{\partial n_{e}\left(\gamma_{e}\right)}{\partial t}+\frac{\partial}{n \gamma_{e}}\left(n_{e}\left(\gamma_{e}\right) \frac{d \gamma_{e}}{d t}\right)=\sum_{i} q_{i}\left(\gamma_{e}\right)
$$

In Equation (24) the terms on the right hand side (r. h. s.) represent sources and sinks of high energy electrons corresponding to production, annihilation, and to processes leading to a sudden loss of a large fraction of the energy of the electron; terms representing leakage out of the halo or the expansion of the universe would also be included on the r. h. s. The factor $d \gamma_{e} / d t$ represents the total gradual energy loss from processes described earlier. We shall consider steady state conditions, so that $\partial n_{e}\left(\gamma_{e}\right) / \partial t=0$.

(i) Electron Spectrum in the Galactic Halo.

From Figure 1 we see that for $\gamma_{e} \leqslant 10^{4}$ (region I) the effective energy loss is primarily by leakage from the halo and the continuity equation reduces to

$$
0=q_{e}\left(\gamma_{e}\right)-n_{e}\left(\gamma_{e}\right) / \tau_{\mathrm{L}},
$$

where $q_{e}\left(\gamma_{e}\right)$ is the production spectrum given by equation (8) and is of the from $k_{e} \gamma_{e}^{-T_{n}}$, and $\tau_{\mathrm{L}}$ is the leakage time. Thus, for $\gamma_{e} \leqslant 10^{4}, n_{e}\left(\gamma_{e}\right)$ is of the form

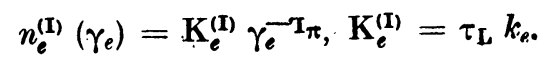


The electron spectrum in region $I$ is essentially the same as the production spectrum, that is, the electrons escape from the galaxy without losing an appreciable amount of their original production energy.

For $\gamma_{e} \geqslant 10^{5}$ (region II) the electrons lose their energy primarily by synchrotron radiation for which $d \gamma_{e} / d t=-b \gamma_{e}{ }^{2}$, and the continuity equation reduces to

$$
-b \frac{\partial}{\partial \gamma_{e}}\left(\gamma_{e}^{2} n_{e}\left(\gamma_{e}\right)\right)=q_{e}\left(\gamma_{e}\right)=k_{e} \gamma_{e}^{-\Gamma_{\pi}}
$$

The solution is then

$$
\begin{gathered}
n_{e}^{(\mathrm{II})}\left(\gamma_{e}\right)=\mathbf{K}_{e}^{(\mathrm{II})} \gamma_{e}^{-\left(\Gamma_{\pi}+1\right.}, \\
\mathrm{K}_{e}^{(\mathrm{II})}=k_{e} / b\left(\Gamma_{\pi}-1\right) .
\end{gathered}
$$

With the assumed values for the parameters and with $k_{e}$ computed from equations (7) and (8) the calculated spectral electron density is shown in Figure 3. The solutions for $n_{e}\left(\gamma_{e}\right)$ in regions I and II were joined smoothly.

\section{(ii) Electron Spectrum in the Intergalactic Medium.}

The approximate spectrum of the intergalactic electrons is calculated by similar procedures. We approximate the effective energy loss for $\gamma_{0} \leqslant 10^{5}$ (region I, see Fig. 1) by the expansion loss and for $\gamma_{e} \geqslant 10^{8}$ (region II) by synchrotron losses. The electron spectrum in the two regions is then given by expressions similar to equations (25) and (27) for the halo, essentially with $\tau_{L}$ replaced by $\tau_{E}$. The calculated spectrum, the curves for the two regions joined "smoothly; is shown in Figure 3 for the previously stated assumed values of the astronomical parameters.

\section{f) High Energy Photon Flux from Various Processes.}

The photon production spectrum by a given process may be computed from the electron (energy) spectrum $n_{e}\left(\gamma_{e}\right)$ and the expression for the photon emission spectrum by this process as a function of $\gamma_{e}$. Denote the photon energy by $\varepsilon$. The energy loss by an electron of energy $\mathbf{E}_{e}$ in time $d t$ due to the emission of $d \mathbb{N}$ photons of energy within $d \varepsilon$ is

$$
-d \mathrm{E}_{e}=\varepsilon d \mathrm{~N}=f\left(\mathrm{E}_{e}, \varepsilon\right) d \varepsilon d t
$$

where $f\left(\mathrm{E}_{e}, \varepsilon\right)$ is the emission spectrum. The number of photons emitted per $\mathrm{cm}^{3}$ per second

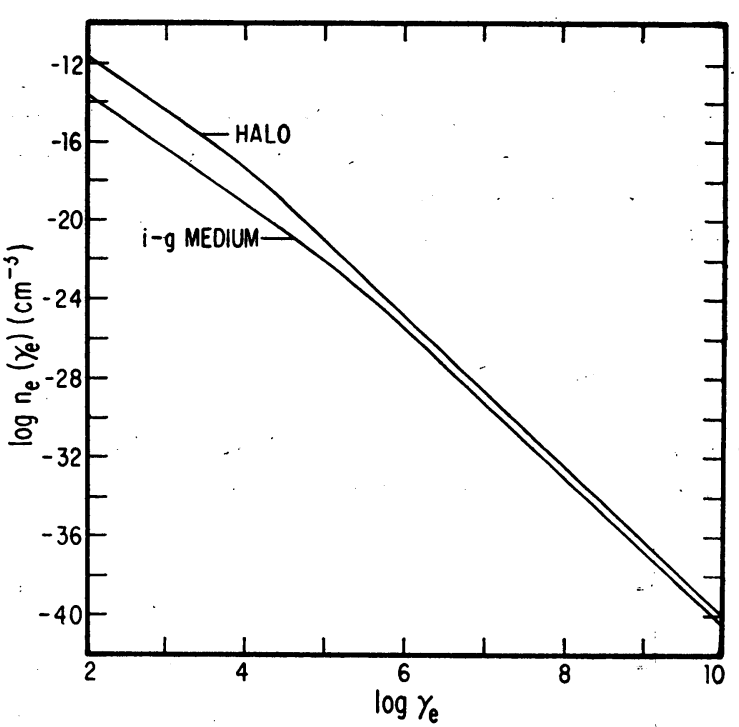

Fia. 3. - Calculated energy spectrum of relativistic electrons in the galactic halo and in the intergalactic medium.

per interval of $\varepsilon$ by an electron spectrum $n_{e}\left(\gamma_{e}\right)$ would then be

$$
\frac{d n}{d t d \varepsilon} \equiv \frac{d n(\varepsilon)}{d t}=\int d \gamma_{e} n_{e}\left(\gamma_{e}\right) \frac{d \mathrm{~N}}{d \varepsilon d t}
$$

We now approximate the emission spectrum by a $\delta$-function at the characteristic photon energy $\varepsilon_{c}$ :

$$
\frac{d \mathrm{~N}}{d \varepsilon d t}=\frac{1}{\varepsilon} f\left(\mathrm{E}_{e}, \varepsilon\right) \rightarrow-\frac{m_{e} c^{2}}{\varepsilon} \frac{d \gamma_{e}}{d t} \delta\left(\varepsilon-\varepsilon_{c}\right),
$$

where $\varepsilon_{c}=\varepsilon_{c}\left(\gamma_{e}\right)$. The photon spectral flux due to emission along a line of sight of path $\int^{\circ} d s=\mathbf{R}$ would be

$$
j(\varepsilon)=\frac{d \mathrm{~J}}{d \varepsilon}=\int \frac{d n(\varepsilon)}{d t} d s=<\frac{d n(\varepsilon)}{d t}>\mathrm{R} .
$$

The incident photon spectra from both the galaxy and the intergalactic medium are readily calculated from the equations (30), (31) and (32) using the derived electron spectra $n_{e}\left(\gamma_{e}\right)$ and the expressions for the energy losses $-\left\langle d \gamma_{e} / d t\right\rangle$. For synchrotron emission $\varepsilon_{c} \approx \hbar \omega_{\mathrm{L}} \gamma_{e}{ }^{2}$; for bremsstrahlung $\varepsilon_{c} \approx m_{e} c^{2} \gamma_{e}$; for the Compton process from electrons with

$$
\gamma_{e} \ll m_{e} c^{2} / \bar{\varepsilon}_{r}, \varepsilon_{c} \approx \bar{\varepsilon}_{r} \gamma_{e}^{2} ;
$$

for the Compton process from electrons with

$$
\gamma_{e} \gg m_{e} c^{2} / \bar{\varepsilon}_{r}, \varepsilon_{c} \approx m_{e} c^{2} \gamma_{e} .
$$

Taking a path length $R=5 \times 10^{22} \mathrm{~cm}$ (the radius of the galactic halo) for the galaxy and a 
path length $R=5 \times 10^{27} \mathrm{~cm}$ (half the Hubble Radius) for the intergalactic medium, the resulting photon spectra are shown in Figure 4. The spectra are for synchrotron, radiation, bremsstrahlung, Compton scattering, and $\pi^{0}$-decay. The spectra from $\pi^{0}$-decay are calculated directly from the pion production spectrum [eq. (7)]. One third of the pions produced are $\pi^{0}$ 's, and each $\pi^{0}$ gives two photons of mean energy $\frac{1}{2} \gamma_{\pi} m_{\pi} c^{2}$. In Figure 4 the photon energy is given with respect to $m_{e} c^{2}$; that is, $\eta=\varepsilon / m_{e} c^{2}, j(\eta)=d j / d \eta$.

The galactic photon fluxes plotted on Figure 4 are averaged over all directions. Actually, the photon flux per steradian from bremsstrahlung and $\pi^{0}$ decay would be greatest in the direction along the galactic plane where the production takes place. The synchrotron radiation and Compton photons would also show a moderate anisotropy due, at least, to our off-center position in the galaxy. We have not computed the spectrum from positron annihilation. The cross section for direct positron (energy : $\gamma_{e} m_{e} c^{2}$ ) annihilation with an electron at rest is, at high energies, (JAUCH and ROHRLICH 1955).

$$
\sigma_{a} \approx \pi r_{0}^{2} \frac{\ln 2 \gamma_{e}}{\gamma_{e}}
$$

so that the bremsstrahlung spectrum dominates the annihilation spectrum by a factor $\sim \alpha \gamma_{e}$ for $\gamma_{e} \geqslant 10^{2}$. At lower positron energies $\left(\gamma_{e}<10^{2}\right)$ ionization losses are dominant (see Fig. 1) and the positron comes essentially to rest before annihilating, giving two photons each of energy $\eta \approx 1$.

To calculate the photon flux from the intergalactic medium we have taken essentially a static Euclidian universe cut off at $R=5 \times 10^{27} \mathrm{~cm}$. It is natural to inquire into the effects of the expansion (differential red shift) and detailed structure of the universe on the resulting photon spectra. It can be shown that only if the photon production spectrum is a power law, will the observed flux show the same shape spectrum (power law with the same index); independent of the structure (including expansion) of the universe. This results essentially because the Doppler-shifted photon energy is proportional to the unshifted energy. As a result, our calculated spectra, which are of the power law type in different energy regions, depend on the detailed structure of the universe only regarding the energy at which the spectra change their slope (at $\eta \sim 10^{5}$ for B and C, Fig. 4). The shift in this critical energy is likely to be less than an order of magnitude, however.

Interstellar and intergalactic photon absorption have also been neglected. Absorption is unimportant except possibly for the long wavelength end of the X-ray region (see GouLd and BurBIDGE 1963) and also for the high energy region around $10^{12} \mathrm{eV}$ in the intergalactic medium At photon energies around $10^{12} \mathrm{eV}$ the absorption cross section for $e^{+}-e^{-}$pair production by (photon-photon) collisions with the thermal quanta of the intergalactic radiation field reaches a maximum (NIKISHEV 1962, see also GOLDREICH and MORRISON 1964). Around this energy the mean free path for photon absorption may be an order of magnitude smaller than the cut-off distance $R \sim 5 \times 10^{27} \mathrm{~cm}$ which we have employed in calculating the photon flux from the intergalactic medium.

We should like to emphasize again that the calculated photon fluxes are only approximate, and this should be kept in mind when we attempt possible interpretations of the observations. In particular, our treatment of meson production in cosmic ray collisions is very rough, especially at low energies where the FERMI theory should be invalid. Moreover, as mentioned earlier, our assumed cosmic ray spectrum is too large at the low energy end ; this effect alone would produce a bend in the calculated photon fluxes at low energies such that the low energy ends of the curves in Figure 4 should be reduced by about an order of magnitude.

\section{g) Comparison}

with Observations - Discussion.

The experimental points exhibited in Figure 4 correspond to the observed cosmic background photon fluxes as summarized in Table I below $\left.{ }^{(}\right)$. The observations are in essentially four energy regions and are over ranges such that $\Delta \eta / \bar{\eta} \sim 1$. There is, of course, another range of energies where cosmic photons are observed, namely, the radio range. The radio spectrum is represented fairly well by the low energy range (not included in Fig. 4) of the calculated synchrotron radiation

(1) In this discussion we have taken the observational values given in Table I at their face value. However, as was stressed by Dr. KraushaAr in his paper at the Liege meeting, it appears now that, while the background $X$-ray fluxes have been detected at the levels quoted, the $\gamma$-ray results are more uncertain and should all be treated as upper limits to the fluxes which may be present. That we are, therefore, only discussing jossible explanations of hypothetical $\gamma$-ray fluxes in this section is to be emphasized. 
TABLE I

THE OBSTRVED HIGH ENERGY COSMIC PHOTON SPEOTRUM

\begin{tabular}{|c|c|c|c|c|}
\hline AUTHOR & $\begin{array}{c}\text { Dinsignation } \\
\text { in Fig. } 4\end{array}$ & EnERGy & $\eta$ & $\begin{array}{c}j(\bar{\eta}) \\
\left(\mathrm{cm}^{-2} \mathrm{~s}^{-1}\right)\end{array}$ \\
\hline - & - & - & - & - \\
\hline GIACOONI et al. (1962) & G & $\sim 2-3 \mathrm{keV}$ & $5 \times 10^{-3}$ & $4 \times 10^{3}$ \\
\hline BowYHR et al. (1964) & B & $\sim 2.3 \mathrm{keV}$ & $5 \times 10^{-3}$ & $2 \times 10^{4}$ \\
\hline ARNOLD et al. (1962) & $\mathbf{A}$ & $\sim 1 \mathrm{MeV}$ & 2 & 0.08 \\
\hline KRAUSHAAR and CuARK (1962) & $\mathrm{K}-\mathrm{C}$ & $\sim 100 \mathrm{MeV}$ & 200 & $4 \times 10^{-5}$ \\
\hline DUTHIE et al. (1963) & $\mathrm{D}$ & $\sim 100 \mathrm{MeV}$ & 200 & $3 \times 10^{-4}$ \\
\hline FIRKowski et al. (1962) & EAS & $\sim 10 \hat{e} \hat{e} \mathrm{eV}$ & $2 \times 10 \hat{e}$ & $10^{-20}$ \\
\hline SUGA et al. (1962) & & & & \\
\hline
\end{tabular}

spectrum. We shall return to this question of the radio spectrum shortly.

We now consider the possibilities of interpreting any of the observed photon fluxes in terms of the various calculated spectra represented in Figure 4. First consider the $\mathrm{X}$-ray observations. The flux $j(\eta)$ for point $\mathbf{X}$ (Fig. 4) is five orders of magnitude above the curve $S^{\prime}$ and six orders of magnitude above $S$. This discrepancy is, in our opinion, sufficient to rule out the interpretation of the point $X$ as due to synchrotron radiation, at least if the high energy electrons are of a secondary origin. The curves $C$ and $C^{\prime}$ do not extend to lower energies because we have considered electrons with $\gamma_{e} \geqslant 10^{2}$, and in our approximate calculations have assumed that

$$
<\varepsilon>_{\mathrm{O}}=\gamma_{\mathrm{e}}^{2} \bar{\varepsilon}_{\mathrm{r}},
$$

giving $\langle\alpha\rangle_{e} \geqslant 30 \mathrm{keV}$. However, due to the distribution of thermal photon energies there is, of course, a distribution of photon energies which can be produced by an electron of given energy. Moreover, for a pion decaying at rest there is still an appreciable probability for a low energy (say, $\gamma_{e} \sim 30$ ) electron being produced. Therefore, the Compton spectra $\mathrm{C}$ and $\mathrm{C}^{\prime}$ certainly do extend to the X-ray region. In spite of this, we do not believe that the $X$-ray point can be due to the Compton process, if the electrons responsible for the scattering have a secondary origin. For, as previously mentioned, the actual cosmic ray spectrum which produces the low energy pions and finally electrons is smaller by about a factor of 10 than the power law spectrum used to compute the curves in Figure 4. A realistic extrapolation of, for example, the curve $\mathbf{C}^{\prime}$ to the $\mathrm{X}$-ray region would still fall about three orders of magnitude below the observational point $B$.

FeLten and Morrison (1963) suggested that not only the $\mathrm{X}$-ray flux, but also the photon fluxes at $\sim 1 \mathrm{MeV}$ and $\sim 100 \mathrm{MeV}$ (see Table II), are due to the Compton process in the intergalactic medium. They suggested that the sources of the high energy intergalactic electrons are the strong radio sources. We can see from the curve $C^{\prime}$ in Figure 4 that the intergalactic spectral density $n_{e}\left(\gamma_{e}\right)$ required to explain the results is about 20-30 times as large as the density which we estimated to result from secondary production in intergalactic space. The Compton spectrum must, of course, extrapolate to the $\mathrm{X}$-ray region and this precludes a secondary origin for the electrons, unless they are produced by a cosmic ray spectrum which has a much higher intensity at low energies than that for cosmic rays observed at the earth. We cannot rule out the FrLTENMorrison hypothesis ; in fact, elementary considerations of the necessary number of sources (radio galaxies) of high energy electrons in the universe suggest that the hypothesis is reasonable quantitatively. As we have shown, for our Galaxy this relatively low energy part of the electron spectrum, that is, the radio electrons, does escape from the galaxy into the intergalactic medium before losing an appreciable amount of their initial energy. As we shall show presently, if the FELTEN-Morrison idea is correct, the amount of synchrotron radiation which these electrons would produce places an upper limit to the intergalactic magnetic field.

Finally, we should like to mention another possible explanation for the observed background 
$\mathrm{X}$-rays. If we assume that the strong discrete $\mathrm{X}$-ray source in Scorpius is at a distance $\sim 10 \mathrm{kpc}$ (roughly the distance to the galactic center), that is, if we assume essentially that it is a galactic source, we can compute its intrinsic $\mathrm{X}$-ray luminosity. If we then assume that on the average every galaxy in the universe has an X-ray source of this luminosity we can compute the background flux from this hypothetical distribution of $X$-ray sources in galaxies out to the Hubble radius. When one performs this elementary calculation (GOULD and BURBIDGE, 1963), one obtains a background flux of roughly the observed magnitude. Clearly, this calculation makes no assumption as to the nature of the individual $X$-ray sources in galaxies, but only that the source in our Galaxy is of average magnitude.

We had originally assumed the strong galactic source to lie in the center of the galaxy. Although the NRL group (BowYER et al., 1964) seems to have established that the strongest $X$-ray source lies in Scorpius, about $20^{\circ}$ off the galactic center, more recent work by both the NRL and MIT groups has indicated that there in indeed a fairly strong $\mathrm{X}$-ray source in the direction of the galactic center. We were led to consider the galactic center as a source of $\mathrm{X}$-ray after performing a theoretical analysis of the apparent excitation conditions in external spiral galaxies (see BurbIdGe, Gould and Pottasch 1963). We found that the stellar ultraviolet radiation in galactic nuclei was insufficient to balance the apparent rapid cooling rate of the interstellar gas in nuclei, and postulated the existence of a flux of corpuscular particles (protons, and/or electrons) in galactic nuclei. Such a particle flux can produce $X$-rays in the interstellar gas either by bremsstrahlung or by $\mathrm{K}$-series emission by certain elements after the ejection of a $\mathrm{K}$-shell electron. HaYaKaWA and Matsuora (1963) have also considered the possible existence of a similar flux of " suprathermal" particles in the galactic spiral arm regions and the associated $X$-ray continuum and line radiation. The particle flux which they envisage is that originally suggested by HAYAKAWA as a heating mechanism in interstellar $H$ I regions. However, this heating source is not really needed to explain the observed temperature of $H I$ regions (see Gould, Gold and SALPETER 1963). Although the hypothetical particle fluxes which were considered by HaYakawa and Kitao and by us may not exist under the original exact conditions, the computed associated X-ray spectra may still be relevant. Quite independent of the specific par- ticle energy spectrum, it appears that the amount of $\mathrm{X}$-radiation emitted in lines ( $\mathrm{K}$-series $\mathrm{X}$-rays) is about $10 \%$ of the integrated continuum radiation. Thus it appears that with only moderate spectral resolution one, may be able to observe cosmic $\mathrm{X}$-ray emission lines.

Regarding the possible interpretation of the observations at $1 \mathrm{MeV}$ (A, Fig. 4), we see that the observed flux is about an order of magnitude above the calculated curve $\mathrm{C}^{\prime}$. In view of the inaccuracies involved this "agreement" within an order of magnitude indicates that Compton scattering by secondary-produced intergalactic electrons provides a possible explanation for the observed photon flux at $1 \mathrm{MeV}$. Of the calculated processes represented in Figure 4 this appears to be the only possible association with the observations at $1 \mathrm{MeV}$. The spectrum from $\pi^{0}$ decay certainly does not extend below $\log \eta=2$ $(E \approx 50 \mathrm{MeV}$ ), and the bremsstrahlung spectra $B$ and $B^{\prime}$ must be less steep below $\log \eta=2$ since, although the energetic secondary electrons can emit a bremsstrahlung spectrum extending to the lower energies, the corresponding bremsstrahlung photon would then carry away only a small fraction of the electron's energy, and the photon production process would be less efficient.

It would appear from Figure 4 that the $\sim 100 \mathrm{MeV}$ photon flux which KraushaAR and CLARK first reported could be accounted for by $\pi^{0^{\prime}} s$ produced in the galaxy or in the intergalactic medium. However, our calculated $\pi^{\circ}$ spectrum, based on the FERMI theory, is very unsatisfactory at the low energy end. For low energy $p-p$ collisions it is primarily $\pi^{+}$mesons that are produced and a more accurate treatment of meson production than our extrapolation of the FERMI theory must be employed. Now, in the KradsHAAR-ClaRK observation the photon flux observed included essentially the whole spectrum from decays of $\pi^{0^{\prime}} s$ of all energies, and most of the $\pi^{0^{\prime}} s$ produced are of low energy. By employing the available data on meson production by incident protons of energy less than $10 \mathrm{BeV}$ and the observed low energy cosmic ray spectrum, Pollack and Fazio (1963) have computed the rate of production of pions by $p-p, p-\alpha$ and $\alpha-p$ collisions per hydrogen nucleus as the rate of production of $\pi^{\circ}$ decay and positron annihilation (after $\pi^{+} \rightarrow \mu \rightarrow e^{+}$decay) photons :

$$
\pi^{0} \text {-decay : } q^{0} \approx 1 \times 10^{-26} \text { photons/s-sterad. }
$$

positron annihilation :

$$
q^{+} \approx 2 \times 10^{-26} \text { photons/s-sterad. }
$$


The $\pi^{0}$-decay photons have energies above about $70 \mathrm{MeV}$ and the galactic positron annihilation photons have energies of about $0.5 \mathrm{MeV}$, since the positrons come essentially to rest before annihilating. The $\pi^{0}$-decay photon flux from a region of density $\left\langle n_{\mathrm{H}}\right\rangle$ of extent $R$ would then be $4 \pi q^{\circ}<n_{\mathrm{H}}>\mathrm{R}$ and in this manner we estimate fluxes of $2 \times 10^{-4}$ photons $/ \mathrm{cm}^{2}$-s and $6 \times 10^{-3}$ photons $/ \mathrm{cm}^{2}-\mathrm{s}$ from the galaxy and intergalactic medium respectively; the galactic flux is a directional average. TheKraUsHAAR and CLARK flux is roughly the same as the calculated contribution from the intergalactic medium while the flux observed by DUTHIE et al. is an order of magnitude larger. The origin of the discrepancy between the KraUshaAR-CLARK and DUTHIE et al. observations may lie in the latter's extrapolation of their balloon observations to zero atmospheric depth. At any rate, it is clear that an upper limit to essentially the product of the intergalactic cosmic ray flux and gas density is established by these observations. The calculated intensity of the positron annihilation line using Poulack and Fazio's value for $q^{+}$and again the "standard" intergalactic gas density $\left(10^{-5} \mathrm{~cm}^{-8}\right)$ is $1 \times 10^{-2}$ photons $/ \mathrm{cm}^{2}-\mathrm{s}$ which is just below the upper limit of $1.5 \times 10^{-2}$ photons $/ \mathrm{cm}^{2}$-s established by ARNold et al. However, intergalactic relativistic positrons do not slow down before annihilating (see Fig. 2) and would not produce a $0.51 \mathrm{MeV}$ line but rather an annihilation continuum extending to higher energies.

The point denoted by EAS in Figure 4 results from observations of Extensive Air Showers (FIRKOWSKI et al. 1962, SUGA et al. 1962) in which an abnormally low number of muons were observed, indicating possibly that the shower was initiated by electromagnetic processes rather than by a nuclear collision. If these showers result from primary photons the flux of these photons would be $\sim 10^{-3}$ times the flux of cosmic ray protons at the same energy. The results of these experiments are questionable and may only represent an upper limit to the primary cosmic photon flux at these high energies. In Figure 4 we see that the EAS point lies 2 or 3 orders sf magnitude above the curve corresponding to the decay of high energy secondary-produced $\pi^{0}$-mesons in the intergalactic medium.

As was mentioned in the footnote at the beginning of this section, it is necessary to emphasize the preliminary nature of all of these observations of high energy photons. While the existence of cosmic X-ray sources seems well established, the

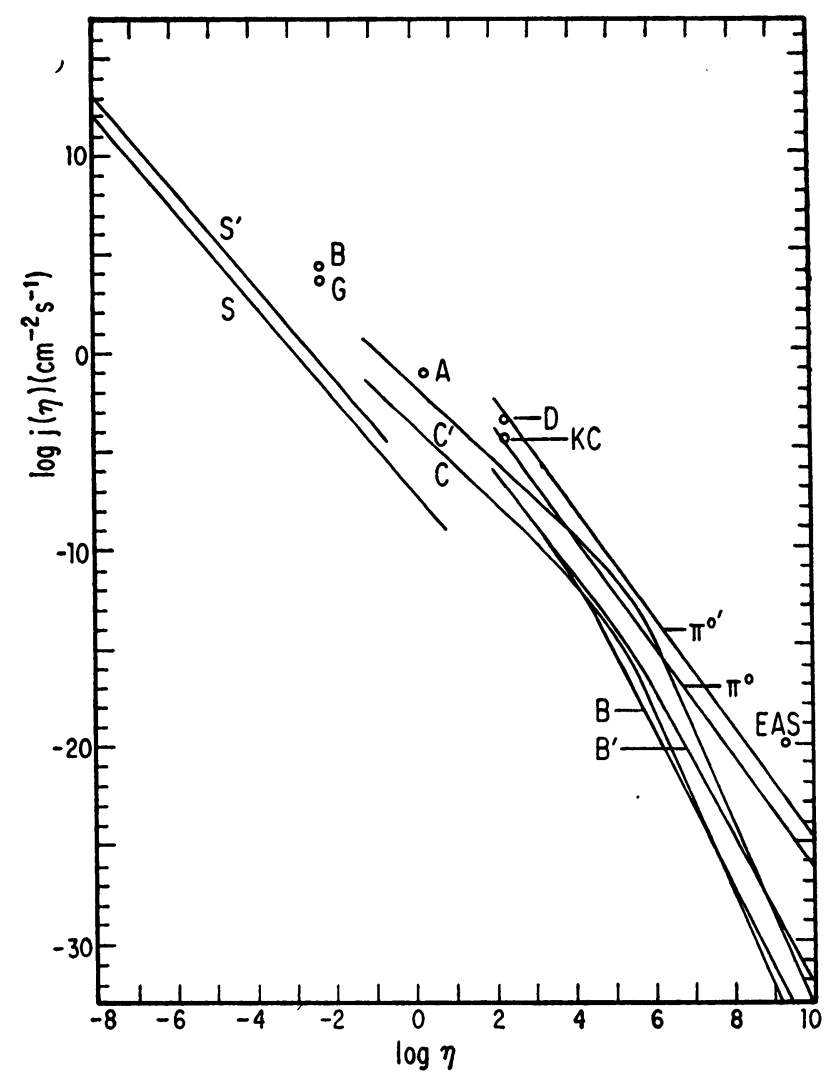

Fig. 4. - Calculated high energy photon background fluxes from synchrotron radiation, Compton scattering, bremsstrahlung, and $\pi^{0}$-decay. The unprimed-designated spectra represent the galactic contributions and the primed denote the spectra from the intergalactic medium. Observational points are denoted by eircles. The letters next to the points refer to the observers (see Table I).

existence of positive fluxes at higher energies (the $\sim \mathrm{MeV}, 100 \mathrm{MeV}$, and $10^{15} \mathrm{eV}$ observations) is not established. The fluxes given for these higher energy photons probably should all be taken as upper limits until the observational situation is clarified. For example, the $\sim \mathrm{MeV}$ observations may be plagued by radioactivity induced in the crystal of the scintillation detector (see Petrerson 1964).

We conclude our discussion here with a few remarks about the observed cosmic radio spectrum from the galactic halo, which is undoubtedly due to synchrotron radiation by relauivistic electrons. We attempt to answer the question as to whether the electron spectrum can be accounted for by secondary production by cosmic rays. This problem has been considered by a number of authors in a manner similar to our treatement. However, our view differs somewhat in that we consider leakage from the halo as the primary loss process for the radio electrons. 
If the energy radiated per second per interval of frequency by an electron of energy $\gamma_{e} m_{e} c^{2}$ is $P\left(v, \gamma_{e}\right)$, the spectral intensity (erg/s-cm ${ }^{2}$-sterad frequency interval) of radiation received from a direction $r$ is

(34) $\quad \mathrm{I}_{\nu}=d \mathrm{E} / d t d \mathrm{~A} d \Omega d \nu$

$$
=(4 \pi)^{-1} \iint n_{e}\left(\gamma_{e}\right) \mathrm{P}\left(\nu, \gamma_{e}\right) d \gamma_{e} d r,
$$

where $n_{e}\left(\gamma_{e}\right) d \gamma_{e}$ is the differential electron density. For an electron spectrum $n_{e}\left(\gamma_{e}\right)=\mathrm{K}_{e} \gamma_{e}^{-\Gamma_{e}}$ the intensity $I_{v}$ may be computed approximately by taking $\mathrm{P}\left(\nu, \gamma_{e}\right)$ to be equal to the expression (13) for $d \mathrm{E}_{e} / d t$ times a $\delta$-function $\delta\left(\nu-v_{\mathrm{L}} \gamma_{e}^{2}\right)$ at the frequency where $P\left(\nu, \gamma_{e}\right)$ is a maximum. Assuming a constant magnetic field $H$ and a path length $\int d r=\mathbf{R}_{h}$, the halo radius, we obtain a familiar result :

$$
\mathrm{I}_{\nu} \approx(12 \pi)^{-1} \quad c r_{0}^{2} \mathrm{~K}_{e} \mathrm{R}_{h} \mathrm{H}^{2} \nu_{\mathrm{L}}^{\left(\Gamma_{e}-3\right) / 2} \nu^{-\left(\Gamma_{\sigma}-1\right) / 2} ;
$$

a power law spectrum with exponent

$$
\alpha=\left(\Gamma_{e}-1\right) / 2
$$

is also obtained using the exact expression for $P\left(\nu, \gamma_{e}\right)$. The constant $K_{e}$ may be determined by the observed value $(500 \mathrm{oK})$ of the radio brightness temperature $T_{b}=I_{v} \lambda^{2} / 2 k$ at $100 \mathrm{MHz}$ in the direction of the galactic pole. Employing equation (35) with $\mathrm{H}=3 \times 10^{-6}$ gauss,

$$
\mathrm{R}_{h}=5 \times 10^{22} \mathrm{~cm}, \quad \Gamma_{e}=2.8(\alpha=0.9),
$$

we obtain $K_{e}=1 \times 10^{-6} \mathrm{~cm}^{-3}$. This number is to be compared with the value calculated from the production and loss processes. By equations (7), (8) and (25) we get for the calculated $\mathrm{K}_{e}$ :

(36) $\mathrm{K}_{e} \approx(8 \pi / 9) \wedge \wedge_{\pi}^{2}\left(m_{\pi} / 4 m_{e}\right)^{\Gamma_{e}-1} \mathrm{~K}_{p}<n_{\mathrm{H}}>\tau_{\mathrm{L}}$.

Using the previously assumed values

$$
\mathrm{K}_{p}=100 \mathrm{~cm}^{-2} s^{-1}, \quad<n_{\mathrm{H}}>=0.03 \mathrm{~cm}^{-3} \text {, }
$$

$\tau_{\mathrm{L}}=3 \times 10^{15} \mathrm{~s}$. we calculate $\mathrm{K}_{e}=1 \times 10^{-6} \mathrm{~cm}^{-3}$; the agreement with the radio value is fortuitous. Actually, the observed radio spectrum has an index $\alpha \approx 0.7-0.8$, and we have adopted the " theoretical" value 0.9 . This discrepancy may not be serious; the observed slightly flatter spectrum could be accounted for by a slight variation with $\gamma_{e}$ of the effective value of $\tau_{\mathrm{L}}$. For example, if $\tau_{\mathrm{L}}$ were slightly shorter for the low energy electrons (caused, perhaps, by another energy loss process at low energy) the smaller value of $\alpha$ and $\Gamma$. could be understood. A more accurate treatment of the production spectrum could also indicate a smaller value for $\alpha$ and $\Gamma_{e}$. Further, we might mention that with our assumed values of the parameters (density, magnetic field, etc...) for the intergalactic medium the calculated synchrotron intensity in the radio region from the intergalactic medium is comparable to that from the halo, whlle, as is seen from Figure 4, the calculated intergalactic synchrotron radiation is actually greater by $\sim 10$ at the high energy end. Admittedly, our calculations are based on many assumptions, but these assumptions may well be valid, and much of the observed non-thermal radio background radiation may be coming from the intergalactic medium.

It is of interest to consider the requirements on the intergalactic magnetic field $\alpha$ if the FrLTENMorrison idea is correct. From Figure 4 we see that for the curve $C^{\prime}$ to pass near the points $X$, $\mathrm{A}$, and $\mathrm{K}-\mathrm{C}$, the value of $\mathrm{K}_{e}$ must be larger by a factor $\sim 30$, or must be $\approx 3 \times 10^{-7} \mathrm{~cm}^{-3}$. One can then compute the intergalactic magnetic field, by equation (35) with $R_{h} \rightarrow 5 \times 10^{27} \mathrm{~cm}$, $\frac{1}{2}$ the Hubble radius, necessary to produce a brightness temperature of $500 \mathrm{oK}$ at $100 \mathrm{MHz}$. One then finds $1 \times 10^{-8}$ gauss for this magnetic field. Thus, if the Felten-Morrison idea is correct, the intergalactic magnetic field must be less than $1 \times 10^{-8}$ gauss.

Finally, we should like to mention a further check on the calculated spectrum of the halo electrons. Recently the French-Italian group (AarinIER et al. 1964) has reported the measurement of a primary cosmic ray electron flux of $6.6 \times 10^{-4}$ particles/ $\mathrm{cm}^{2}$-s-sterad for $\mathrm{E}_{e}>4.5 \mathrm{BeV}$, corresponding also to an electron/ proton cosmic ray ratio of $1 \times 10^{-2}$. This measurement of the primary electron flux at fairly high energies is probably more reliable than results of measurements at lower energies which are influenced by solar activity. The measured flux is to be compared with that from the calculated spectra above

$$
4.5 \mathrm{BeV}\left(\gamma_{e}>\gamma_{0}=4.5 \mathrm{BeV} / m_{e} c^{2}\right) \text {. }
$$

One finds, with $\mathrm{K}_{e}=1 \times 10^{-6} \mathrm{~cm}^{-3}, \Gamma_{e}=2.8$, a flux

$$
(4 \pi)^{-1} \int_{\gamma_{0}}^{\infty} c K_{e} \gamma_{e}^{-\Gamma_{e}} d \gamma_{e} \approx 1 \times 10^{-4}
$$

particles $/ \mathrm{cm}^{2}$ s-sterad.

This flux is somewhat smaller than the observed one, but in view of the uncertainties involved in 
the calculations, agreement within an order of magnitude is all that one could hope for.

\section{h) The Hot Universe Model - Bremsstrahlung from the Intergalactic Medium.}

GoLD and HoyLe (1958) have suggested a cosmological model in which the intergalactic medium is at a very high temperature $\left(\sim 10^{9} \mathrm{KK}\right)$. The high temperature is supposed to arise from the $\sim 1 \mathrm{MeV}$ electrons which would result after the decay of spontaneously created neutrons as envisioned in the steady-state theory. Galaxy formation within the framework of this model was considered by Burbidas, BURBIDGe, and HOYLE (1963). An observational test of this model can be made, since such a hot intergalactic medium would emit thermal bremsstrahlung photons in the $\mathrm{X}$-ray region where observations have been made (BowYER et al. 1964). For a mean thermal electron energy $\left\langle\mathrm{E}_{e}\right\rangle=50 \mathrm{KeV}$, and a density $n_{e}=n_{p}=1.2 \times 10^{-5} \mathrm{~cm}^{-3}$ the rate of production of bremsstrahlung photons within the energy range of the observations is about $r_{b}=1.17 \times 10^{-25}$ photons $/ \mathrm{cm}^{3}$-s (Gould and Burbidae 1963). Taking a cut-off radius $R=5 \times 10^{27} \mathrm{~cm}$ for the universe, one calculates a flux $f_{b}=r_{b} \mathbf{R} / 4 \pi \sim 50$ photons $/ \mathrm{cm}^{2}$-s-sterad to be expected at the earth. This flux is $\sim 10$ times the observed $\mathbf{X}$-ray background flux and is evidence against the hot universe model (and the steady-state theory with spontaneous creation of neutrons). Actually, if the appropriate intergalactic density to be used is four times the usually adopted $2 \times 10^{-26} \mathrm{gm} / \mathrm{cm}^{3}$, as suggested by ScIAMa (1964), the disagreement with observations is even more violent. In any case it appears that the $\mathrm{X}$-ray observations have established an upper limit of $10^{7} \mathrm{~K}$ for the temperature of the intergalactic medium.

\section{i) Matter and Anti-Matter and the Steady \\ State Cosmological Theory.}

The attractive feature of the steady state is its simplicity. The unique feature is a spontaneous creation rate of " new" matter $d n / d t \sim 3 \mathrm{H} n$, where $n \sim 10^{-5} \mathrm{~cm}^{-3}$ is the mean matter density in the universe (taken to be the mean hydrogen density in the intergalactic medium) and $\mathrm{H}$ is the Hubble constant $\left(3 \mathrm{H} \sim 10^{-17} \mathrm{~s}^{-1}\right)$. One might expect that in the spontaneous creation process, to conserve baryon and lepton number, particles and antiparticles are created. Since the expansion rate constant $3 \mathrm{H}$ is about two orders of magnitude greater than the annihilation rate (see below), BURBIDGE and Hoyle (1956) suggested the possibility of an appreciable abundance of anti-matter in the universe. This idea can be put to a test, since the end products of matter and antimatter annihilation are observable high energy $\gamma$-rays.

Let us suppose that $\left(p, e^{-}\right)$and $\left(p, e^{+}\right)$are spontaneously produced and have a steady state mean number density $n=10^{-5} \mathrm{~cm}^{-3}$ and $\alpha n$ respectively, where $\alpha$ denotes the mean ratio of anti-matter to matter (or vice-versa). The electron-positron annihilation cross section at nonrelativistic energies is ( $\mathrm{J}_{\mathrm{AUCH}}$ and ROHRLICH 1955) $\sigma_{a}=\pi r_{0}^{2} / \beta$, where $r_{0}$ is the classical electron radius and $\beta=v / c$. The annihilation rate is then

$$
d n_{a} / d t=\bar{\alpha} n^{2} \pi r_{0}^{2} c \sim \bar{\alpha} \times 10^{-24} \mathrm{~cm}^{-3} \mathrm{~s}^{-1},
$$

and the expected flux of $0.51 \mathrm{MeV}$ photons from the intergalactic medium out to a distance $\mathrm{R} \sim 5 \times 10^{27} \mathrm{~cm}$ is $2 \mathrm{R} d n_{a} / d t \sim \alpha \times 10^{4}$ photons $/ \mathrm{cm}^{2}$-s. This can be reconciled with the upper limit of $10^{-2}$ photons $/ \mathrm{cm}^{2}$-s suggested by ARNold et al. (1962) only if $\bar{\alpha}<10^{-6}$. This means that if there is appreciable anti-matter in the universe, it must be separated from matter, so that it cannot annihilate and produce observable $\gamma$-radiation.

A limit on the amount of anti-matter in the universe can also be provided from an analysis of the $\gamma$-ray experiments at higher energies which can detect $\pi^{0}$-decay $\gamma^{\prime} s$. In the proton-antiproton annihilation $\sim 5$ pions are produced, some of which are $\pi^{0^{\prime}} s$ which produce $\gamma$-rays of energy $\sim 100 \mathrm{MeV}$ in their decay. In each $p-p$ annihilation about $4 \gamma$-rays are produced. Taking an annihilation cross section

$$
\pi \Lambda_{\pi}^{2} \sim 6 \times 10^{-26} \mathrm{~cm}^{2}
$$

and a mear relative $p-p$ velocity ${ }^{1}$ ) of $\sim 10^{7} \mathrm{~cm} / \mathrm{s}$, one computes a flux of $\sim 100 \mathrm{MeV} \gamma$-rays of $\sim \bar{\alpha}$ photons $/ \mathrm{cm}^{2}$-s. The experiments of KRAUSHAAR and Clark and DUthise et al. would then imply $\bar{\alpha} \leqslant 10^{-2}$. Thus, it appears that in the steady state cosmology matter and anti-matter

(1) This corresponds to a thermal velocity at $\sim 10^{5}$ o. K which is the latest estimate of the temperature of intergalactic matter (Scrama, 1964a). The relativistic electrons and positrons from the decay of charged pions produced in $p-p$ annihilations would not be thermalized in a time $(3 \mathrm{H})^{-1}$ and would produce a weak annihilation continuum. 
cannot be created in comparable amounts in the same region.

Finally, regarding cosmological tests, we should like to mention the recent discussion by ScIAMA ((1964b; see also Gould and Scrama 1964). SCIAMA indicates how the measurement of the shape of an emission line, smeared into a continuum by the cosmic differential red shift, would provide information about the structure of the universe at great distances.

\section{High energy RAdiation from stars.}

\section{a) Hard Radiation from Stellar Coronae.}

Since the Sun is the only star whose corona is directly detectable, all theories concerning the origin and conditions in a corona have stemmed from it. The first question that arises is therefore whether it is plausible to suppose that other stars have coronae similar to that of the Sun. To answer that question it is necessary to consider the probable origin and source of heating of the solar corona. The theory of the expanding solar corona (cf. PARKER, 1963) is based on the concept that the convection below the photosphere generates wave motion (both acoustic and hydromagnetic waves have been discussed) which propagate upward and dissipate, and it is the dissipative heating which leads to coronal expansion. It therefore may be supposed that all stars which have extensive outer convection zones will maintain expanding coronae. This would imply that all main sequence stars below about

$$
\mathrm{F} 2\left(\mathrm{M} \leqslant 1.5 \mathrm{M}_{\odot}\right)
$$

would have extensive coronae and these stars comprise a considerable fraction of the mass of a galaxy. Also, all stars in the giant stage of their evolution would have coronae. The critical question next is to estimate the average temperature of such hypothetical coronae.

PARKer (1963) has pointed out that coronae heated at their bases will have temperatures given approximately by the relation $\mathbf{G M} m_{\mathrm{H}} / \mathrm{RsT} \geqslant 4$, or $\mathrm{T} \leqslant 5.8 \times 10^{6}(\mathrm{M} / \mathrm{R}){ }^{\circ} \mathrm{K}$ with $(\mathrm{M} / \mathrm{R})$ measured in solar units.

For stars on the main sequence $M / R$ is of the order unity so that coronal temperatures in the range $10^{6}-10^{7}$ degrees are to be expected. For giant stars $M / R$ is $\leqslant 0.1$ and for supergiants it is $\leqslant 0.01$. Thus the temperatures of the hypothetical coronae of giant are expected to be $\leqslant 10^{6}$ degrees, while for super-giants they are $\leqslant 10^{5}$ degrees, and it would appear that only main sequence stars are likely to have hot enough coronae to emit $\mathrm{X}$-rays. PArker has given various arguments for supposing that more massive main-sequence stars also may have coronae. However, the spectroscopic evidence for extended atmospheres in these stars suggests that the gas has temperatures only $\rightarrow 10^{4}$ degrees (the heating is by dilute stellar radiation). Thus it is highly improbable that they have hot coronae.

It is of interest to estimate the expected X-ray flux from the coronae of all the stars in the galactic disk. The problem of estimating this flux, made difficult by our lack of knowledge of the density, temperature, and volume of stellar coronae, was considered by Wallace TuCker at UCSD. We shall outline his calculations briefly and present the results. The $\mathrm{X}$-ray emission from a hot corona in the 2-8 $\AA$ region (where there are observations) is produced principally by bremsstrahlung, radiative recombination, and by line emission following $\mathrm{K}$-shell electron collisional excitation. In computing the fluxes due to thèse processes the methods employed by ELWERT (1952, 1954, 1961) were used, whereby the ions are assumed to be hydrogen-like and proper correction factors (Gaunt factors) are introduced when necessary. The power radiated by each element is proportional to the product $n_{i} n_{e}$ of the electron and ionic density, so that the ionization equilibrium $\left(n_{i} / n_{e}\right)$ must be computed. This has been done by House (1964) for the elements from $H$ to $F e$ for temperatures up to 11 million degrees, using the classical ionization theory developed by ELwERT and others. The values of $n_{i} / n_{e}$ arrived at in this manner by House and the element abundances for the solar corona as determined by POTTASCH (1960) were used to compute the 2-8 $\AA$ X-ray power $\mathrm{P}_{x}$ radiated per unit volume by a MAXWELLIAN gas for different temperatures given in Table II. The bremsstrahlung is due mainly to hydrogen while the recombination and line radiation are produced predominantly by other abundant light elements such as $\mathrm{He}, \mathrm{C}, \mathrm{O}, \mathrm{Mg}$, $\mathrm{Si}, \mathrm{S}$, and Fe.

BuRgess and Seaton (1964) have pointed out that the inclusion of (radiation-less) dielectronic recombination increases the recombination rate significantly $(\sim 20$ times $)$ for iron at coronal densities. Since dielectronic recombination can occur in any ion with an autoionizing level sufficiently close to the bound levels and possessing a large probability for a transition to some bound level, it appears that the ionization equilibrium 
calculations of Houss must be revised. The net effect is to depress the degree of ionization, and to reduce the power radiated by recombination and line emission, since the bulk of the radiation from these processes in the $2-8 \AA$ region comes from highly ionized atoms. For this reason the numbers given in Table II for recombination and line emission must be viewed as upper limits to the actual rates which are probably closer to the bremsstrahlung contribution.

\section{TABLE II}

\section{POW RR RADIATED BY A MAXWELLIAN GAS}

\begin{tabular}{|c|c|c|c|}
\hline \multirow[t]{2}{*}{$\mathbf{T}_{e}\left({ }^{\circ} \mathrm{K}\right)$} & \multicolumn{3}{|c|}{ 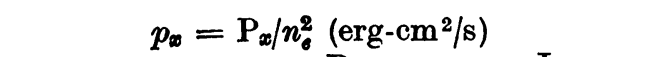 } \\
\hline & BREMSSTRAHLUNG & $\begin{array}{l}\text { RECOM- } \\
\text { BINATION }\end{array}$ & $\begin{array}{c}\text { LINE } \\
\text { RADIATION }\end{array}$ \\
\hline- & - & - & - \\
\hline $10^{6}$ & $9 \times 10^{-32}$ & $3 \times 10^{-30}$ & $7 \times 10^{-31}$ \\
\hline $2 \times 10^{6}$ & $5 \times 10^{-28}$ & $8 \times 10^{-27}$ & $4 \times 10^{-27}$ \\
\hline $4 \times 10^{6}$ & $6 \times 10^{-26}$ & $1 \times 10^{-25}$ & $1 \times 10^{-25}$ \\
\hline $6 \times 10^{6}$ & $3 \times 10^{-25}$ & $4 \times 10^{-25}$ & $2 \times 10^{-24}$ \\
\hline $8 \times 10^{6}$ & $6 \times 10^{-25}$ & $8 \times 10^{-25}$ & $3 \times 10^{-24}$ \\
\hline $10^{7}$ & $1 \times 10^{-24}$ & $1 \times 10^{-24}$ & $3 \times 10^{-24}$ \\
\hline
\end{tabular}

Frmbdan (1961) has made several measurements of the X-ray emission of the Sun. Taking the largest flux measured for the quiet corona $\left(\sim 10^{4}\right.$ counts $\left./ \mathrm{cm}^{2}-\mathrm{s}\right)$, a temperature of $\mathrm{T}_{c} \sim 4 \times 10^{6} \mathrm{oK}$ and an integrated electron density of $\int n_{e}^{2} d \mathrm{~V}_{c} \sim 6 \times 10^{48} \mathrm{~cm}^{-3}$ are required for agreement between theory and observation. The contribution to the volume integral is thought to come from condensations whose density is comparable to that of the chromosphere. The question arises as to how to fix $\int n_{e}^{2} d \mathrm{~V}$ and $\mathrm{T}$ for coronae of other stars. If the volume is taken to be a constant fraction of the stellar volume, then $V \propto R^{3}$, where $R$ is the stellar radius. The density can be established roughly (de JAGER 1960) by setting the amount of mechanical energy in acoustic waves fed into the chromosphere per unit area per unit time $\left(\propto \rho V_{s}^{8}\right.$, where $\rho=n_{s} m_{\mathrm{H}}$ is the density and $\mathrm{V}_{s} \propto \mathrm{T}_{s}^{1 / 2}$ is the sound velocity $; T_{1}=$ chromospheric temperature $\approx$ surface temperature) equal to the amount of energy $\left(\propto \mathrm{Hn}_{e}^{2} \mathrm{~T}_{s}^{-12}\right.$ ) radiated in recombination in a chromospheric gravitational scale height $\mathrm{H}\left(\propto \mathrm{T}_{s} \mathrm{R}^{2} / \mathrm{M}, \mathbf{R}=\right.$ stellar radius, $\mathrm{M}=$ stellar mass $)$. Then $n_{e} \propto \mathrm{T}, \mathrm{M} / \mathbf{R}^{2}$, and $n_{e}^{2} \mathrm{R}^{3} \propto \mathrm{T}_{s}^{2} \mathbf{M}^{2} / \mathbf{R}$.
In this manner $\int n_{e}^{2} d V$ was determined from $T_{s}, M$ and $R$ for all stars by normalizing to the value $\left(6 \times 10^{48} \mathrm{~cm}^{-3}\right)$ for the Sun. The coronal temperature was assumed proportional to $M / R$ and was also normalized to the assumed solar value $4 \times 10^{6} \mathrm{oK}$.

To calculate the $\mathrm{X}$-ray flux $\mathrm{F}_{\infty}$ to be expected from all stars in the galactic disk one must sum over all stars in the volume $V_{a}$ of the disk. This is accomplished by introducing the main sequence luminosity function $\varphi\left(\mathbf{M}_{v}\right)$ which gives the number of stars per cubic parsec with absolute visual magnitude between $M_{v}-\frac{1}{2}$ and $M_{v}+\frac{1}{2}$. It was assumed that $\varphi$ is constant throughout the galactic disk except in the $z$-direction that is,

$$
\varphi\left(r, \mathrm{M}_{v}\right)=\varphi\left(z=0, \mathrm{M}_{v}\right) \exp (-z / \beta),
$$

where $\varphi\left(z=0, M_{v}\right)$ is the luminosity function for the solar neighborhood and $\beta=\beta\left(\mathbf{M}_{0}\right)$. Using the data on $\mathbf{T}_{8}\left(\mathbf{M}_{v}\right), \mathbf{M}\left(\mathbf{M}_{v}\right), \mathbf{R}\left(\mathbf{M}_{v}\right)$ and $\beta\left(\mathbf{M}_{v}\right)$ given by Alume (1963) one computes, neglecting galactic absorption (cf. GoULD and BURBIDGE 1963), a flux

$$
\begin{aligned}
\mathbf{F}_{x} & =\iiint d \mathrm{~V}_{0} d \mathrm{M}_{v} d \mathrm{~V}_{\mathbf{L}}\left(4 \pi r^{2}\right)^{-1} p_{x}\left(\mathrm{M}_{v}\right) \varphi\left(\mathbf{M}_{v}\right) \\
& \approx 4 \times 10^{-11} \mathrm{erg} / \mathrm{cm}^{2}-s
\end{aligned}
$$

with most of the contribution coming from the hotter stars with $\mathbf{M}_{v}<0$ for which

$$
\mathrm{T}_{\mathrm{c}} \sim 6-8 \times 10^{6} \mathrm{oK} \text {. }
$$

If, as previously mentioned, only the cooler stars have coronae, then the flux from stars with say, $\mathbf{M}_{v}>\mathbf{3}$ is about an order of magnitude less. We might mention that, if one assumes a constant value of $\int d \mathrm{~V}_{c} n_{\theta}^{2}$ for all stars fixed by the solar value, the flux from stars with $-\infty<\mathrm{M}_{v}<\infty$ is about $1 / 10$ as large as (37) with the main contribution now coming from the cooler stars.

These calculations are for quiet coronae. Measurements have been made of the enhancement of the solar X-ray flux as a result of flares. The mean measured flux for the disturbed corona was found to be enhanced by a factor $\sim 400$ (Aller 1963, Friedman 1961, see also part $b$ of this Sect.). Of course,-there is no reason to suppose that the solar corona is typical but it is conceivable that such an enhancement can occur in the coronae of other stars. All known uncertainties considered, we would estimate that the expected X-ray flux from the coronae of all stars in the galactio 
disk to lie within $10^{-12}-10^{-8} \mathrm{erg} / \mathrm{cm}^{2}-\mathrm{s}$. The observed background intensity is

$$
4 \times 10^{-8} \mathrm{erg} / \mathrm{cm}^{2}-\mathrm{s} \text {. }
$$

(BOWYER et al. 1964). It appears that although it is unlikely that stellar coronae produce a significant X-ray flux, they cannot definitely be ruled. out.

\section{b) Hard Radiation \\ Emitted Following Violent Activity of Stellar Surfaces.}

We now turn to the possibility that temporary violent activity gives rise to fluxes of detectible hard radiation from stars. Apart from stellar explosions which will be discussed later, this acti vity is often manifested through a mechanism which generates an outburst of high-energy particles on the star's surface. These particles, by one or several of the processes described earlier, will give rise to energetic photons. Again the only method of estimating the fluxes is to consider what is observed from the Sun. As has been described by FrIedman (1961) flare activity gives rise to a great enhancement of the $\mathrm{X}$-ray flux (Table VII of FrIedMaN). It appears that the radiation is increased because the temperature is increased and also because a flux of high-energy particles (as manifested by the Type IV radio burst) is generated.

From FrIEDMax's table we see that the enhancement of the X-ray flux amounts to a factor $\sim 10^{5}$ at times of maximum flare activity so that the maximum solar $\mathrm{X}$-ray luminosity is $\mathrm{L}_{x} \leqslant 10^{26} \mathrm{erg} / \mathrm{s}$. We may therefore consider what the maximum intensities of the fluxes that we might expect from stars could be. We take three representative cases :

a) A single solar-type star at distance $d=1$ pc.

b) A galactic star cluster (100 members reaching maximum flare activity at the same time) at distance 100 pc.

c) $10^{10}$ stars near the galactic center all at maximum flare activity at a distance of $10^{4} \mathrm{pc}$.

The fluxes $\mathrm{L}_{x} / 4 \pi d^{2}$ to be expected in such circumstances are

$$
10^{-12}, 10^{-14} \text {, and } 10^{-10} \mathrm{erg} / \mathrm{cm}^{2}-\mathrm{s}
$$

for cases $a, b$ and $c$ respectively. All of these fluxes are appreciably smaller than the flux $\sim 10^{-7} \mathrm{erg} / \mathrm{cm}^{2}-\mathrm{s}$ detected from the Scorpius $\mathrm{X}$-ray source by BowYER et al (1964).

Radiation harder than a few $\mathrm{KeV}$ can only be emitted from a star's surface in nuclear reactions, by bremsstrahlung, the synchrotron mechanism, or possibly by the Compton process. Donlan and Fazio (1964) have recently given a fairly extensive discussion of the processes which may be expected to produce $\gamma$-rays from the Sun. From an observational point of view only $\gamma$-rays with energies $\leqslant 0.5 \mathrm{MeV}$ have so far been detected from the Sun (Perterson and WinkLer 1959) and this during a flare. The flux that they observed from a $\gamma$-ray burst was $\sim 30$ photons $/ \mathrm{cm}^{2}$-s of energy $\sim 0.5 \mathrm{MeV}$. If we use this as a standard to make estimates of the fluxes from the representative aggregates $a, b$ and $c$ above we obtain $10^{-9}, 10^{-11}$ and $10^{-7}$ photons $/ \mathrm{cm}^{2}$-s respectively. It appears that these fluxes are too weak to be observed. Nuclear reactions undoubtedly occur in solar flares but again it is exceedingly difficult to make estimates of the final products or the total fluxes of hard radiation which may be expected here, because of the uncertainty in the total flux of particles which is nresent in a flare.

\section{c) Neutron Stars.}

It has been pointed out by CHro (1964) and Frnzi (1964a) that, since it is possible that neutron configurations may be reached as an end phase of stellar evolution by processes which leave the star extremely hot, such configurations may, for rather a short period, be thermal X-ray emitrers. However, from the theoretical standpoint it must be conceded that at the present time we cannot demonstrate conclusively that stable neutron configurations are ever formed or can exist if formed. The presumption of these authors is that the neutron configurations are formed during a supernova outburst, as was first proposed by BAADI and ZwICKY (1938) and ZWICKY (1938) many years ago. Since there has been a distinct tendency in the recent literature to suggest rather strongly that the observations of X-ray sources in Scorpius and in the vicinity of the Crab show that neutron stars do exist, it is probably worthwhile mentioning some of the theoretical uncertainties associated with neutron configurations. The observational uncertainties will be discussed in the concluding section.

It is well known that there is a critical mass for a degenerate neutron configuration above which no stable equilibrium is possible. This result was first derived by LANDAU (1932) and calculations by OPPENHEIMER and VolKOFF (1939) gave a value of about $0.7 \mathrm{M}_{\odot}$ for this observable mass limit. While in later calculations this mass limit 
has been slightly revised, it is clear that the mass limit lies near $1 \mathbf{M}_{\odot}$. Even the doubtful assumption of a hard-core nuclear potential, which is known to be incorrect from relativistic considerations, only extends the maximum mass to about $3 \mathbf{M}_{\odot}$. In fact it is clear from the earliest considerations (cf. LANDAU 1932) that the maximum mass is very insensitive to the equation of state at nuclear densities and above. For masses above the critical mass it appears that implosion must occur (DATT 1938; OPPENHELMER and SNyder 1939). For a modern review see Hoyle, Fowler, Burbidat and BURBIDGe (1964). Thus, if neutron configurations which can exist long enough to be detected as sources of $\mathrm{X}$-rays come from supernova outbursts, it is required that in the supernova outburst sufficient mass is ejected so that the resulting configuration falls below the limit for support by a degenerate neutron configuration. None of the attempts to unravel the processes of supernova outbursts have yet given any real indication that such conditions can be achieved. The attempts by the California-Cambridge group (BURBIDGE, BURBIDGE, FowLER, and HOYLE 1957 ; FOWLER and HOYLE 1960 ; Fowler and Hoyle 1964) have not been able to answer this question. Even the range of masses of stars which become supernovae is in doubt, but it appears highly probable that the Type II supernovae are stars of quite large mass $\sim 30 \mathrm{M}_{\odot}$ (cf. Fowler and HoYLe 1964). All of the discussion of the supernova outbrust as it applies to the last phases of nucleosynthesis, and neutrino emission, etc... have been carried out by neglecting the effects of rotation. However, as has been shown by HoYLE et al. (1964) this may have the effect of allowing a massive star to fragment, either into white dwarf ,or into neutron configurations (cf. Equation (45) of that paper). In the work of CHrU (1964) no conclusion as to whether a degenerate neutron configuration with a mass below the critical mass is left has been reached. The only attempt at a hydro-dynamical calculation of the implosion of a supernova before relativistic effects become important is that by CoLGATE and his colleagues (1963). This calculation follows the collapse until nuclear densities are reached, but then it is supposed that a bounce occurs and the outer envelope is ejected. The calculation is not able to determine what fraction of the mass is left as a degenerate neutron configuration.

The only supernova remnant which can be studied in any detail is the Crab Nebula. While there are uncertainties in the mass of the nebula, analysis shows that it is only $\sim 0.64 \mathrm{M}_{\odot}\left(\mathrm{O}^{\prime} \mathrm{DELL}\right.$, 1962) so that if the outburst originated from a star with a mass in excess of about $3 \mathbf{M}_{\odot}$ (and the type of supernova involved is still uncertain, as is the relation of type with mass) it must be concluded either that a large remnant has imploded or else that it is fragmented into a number of neutron stars.

Finally there is some question about the stability of neutron configurations. The question of their dynamical stability has recently been considered for a range of models by Misner and ZAPOLSKY (1964) who have concluded that dynamically stable solutions exist for stars below the maximum mass for cold static equilibrium. With these uncertainties in mind we turn to the calculations of the fluxes of X-rays which may be emitted by neutron stars. Such calculations have been made by CHIU and SALPETER (1964) and MorTON (1964). The calculations are in fairly good agreement and we reproduce as Table III the table of CHIU and SALPETER giving the emission characteristics of a $0.5 \quad M_{\odot}$ neutron star of radius $10 \mathrm{~km}$ for various values of the core temperature $\left(^{1}\right)$.

We shall not discuss here the recent very beautiful lunar occultation $\mathrm{X}$-ray observations (Friedman 1964) of the Crab Nebula and the associated interpretation. These results are to be found in a paper presented at this Symposium.

\section{Supernova Remnants.}

Of the possible sources of energetic photons and neutrinos in the Galaxy one is led immediately to consider the supernova remnants. Hard radiation may be emitted during and immediately after a supernova explosion, and since large fluxes of relativistic particles are known to be present in supernova remnants for periods of $\leqslant 1000$ years after the explosions occurred, fluxes of hard photons may be generated for longer times.

We consider briefly the hard radiation that may be emitted at the time of the explosion, and then

(1) Very recently Finzi (1964b) and SALPETER (private communication) have concluded that, while the neutrino emission rates which determine the lifetime of a neutron star are still rather uncertain, the time scales may have been overestimated in the work of CHIU and SALPETER and may only be of order $1-10$ years. If this is the case then the chance of detecting a neutron star by means of its $X$-ray emission while it is still hot is very small, and $\mathrm{X}$-rays from the CrAB cannot have come from such a source. 
TABLE III

FLUX FRom A NEUTRON STAR OF 0.5 $\mathrm{M}_{\odot}$

(Taken from ChIU and SALPETER)

\begin{tabular}{|c|c|c|c|c|c|}
\hline $\begin{array}{l}\text { Core Température } \\
(\mathrm{oK})\end{array}$ & $\begin{array}{c}\text { Absolute Luminosity } \\
\text { (photons) } \\
\text { erg/s }\end{array}$ & $\begin{array}{l}\text { OPтTMUM } \lambda \\
\text { (A) }\end{array}$ & $\begin{array}{c}\text { FLUX } \\
\text { (erg/s) } \\
\text { DisTANT } \\
100 \text { pe }\end{array}$ & $\begin{array}{c}\text { FuUx } \\
\text { (erg/s) } \\
\text { DistaNt } \\
1000 \text { pc }\end{array}$ & $\begin{array}{c}\tau \\
\text { (YEARS) }\end{array}$ \\
\hline - & - & - & - & - & - \\
\hline $1 \times 10^{9}$ & $8 \times 10^{-6}$ & 3.1 & $7 \times 10^{-6}$ & $7 \times 10^{-8}$ & 1000 \\
\hline $5 \times 10^{8}$ & $3 \times 10^{6}$ & 3.9 & $2.5 \times 10^{-6}$ & $2.5 \times 10^{-8}$ & 1000 \\
\hline $2 \times 10^{8}$ & $3 \times 10^{-5}$ & 6.8 & $2.5 \times 10^{-7}$ & $2.5 \times 10^{-9}$ & 1500 \\
\hline
\end{tabular}

consider in more detail the conditions in the Crab nebula.

Present theory of supernova outbursts makes no detailed prediction of the flux to be expected, but two processes may be important. These are :

a) Nuclear $\gamma$-rays emitted in the process of nucleosynthesis at the time of the outburst.

b) $\gamma$-rays emitted through the early interaction of a cloud of relativistic particles with the magnetic field and material in the expanding shell.

a) If, in a supernova outburst the inner part implodes and the outer part is suddenly heated so that hydrogen burning takes place very rapidly, we can suppose that the bulk of the energy released is degraded through its passage through the material, but some fraction, perhaps the energy released in burning $0.01 \mathrm{M}_{\odot}$ of hydrogen, will be emitted as $\gamma$-rays in the $\mathrm{MeV}$ range. Thus we might suppose that $10^{50} \mathrm{ergs}$ is emitted in $\sim 1000$ seconds. For a galactic supernova at assumed distances of 1 and $10 \mathrm{Kpc}$ this gives fluxes at the earth of $10^{3}$ and $10^{5} \mathrm{erg} / \mathrm{cm}^{2} \mathrm{~s}^{-1}$, fantastic rates. However, the appearance of a galactic supernova is highly improbable. From extragalactic supernovae at characteristic distances of 10 and $100 \mathrm{Mpc}$ the fluxes would be $10^{-5}$ and $10^{-7} \mathrm{erg} / \mathrm{cm}^{2} \mathrm{~s}^{-1}$ respectively. These rates are obviously uncertain by several powers of 10 . at might also be expected that some part of the flux is degraded to the energies of a few kilovolts and is emitted as X-rays: As an upper limit we might suppose that this flux is of comparable intensity for a few days with the flux at maximum light from the supernova. If we suppose that it reaches a value of $M_{r}=-18$ this corresponds to $10^{43} \mathrm{erg} / \mathrm{s}$ and at distances of $1 \mathrm{Kpc}$ and $10 \mathrm{Kpc}$
(Galactic) and $10 \mathrm{Mpo}$ (extragalactic) fluxes at earth of $10^{-1}$ and $10^{-3} \mathrm{erg} / \mathrm{cm}^{2} \mathrm{~s}^{-1}$ (Galactic) and $10^{-9}$ and $10^{-11} \mathrm{erg} / \mathrm{cm}^{2} \mathrm{~s}^{-1}$ may be expected.

b) A large flux of relativistic electrons is currently present in many supernova remnants, and it is possible that this in part is the remnant of a much larger flux of relativistic particles which was produced at the time of the outbursts. Let us suppose that some $10^{50} \mathrm{erg}$ of particles, largely protons, was generated in the explosion. If they are originally confined in an expanding shell containing a magneric field (they are the relativistic plasma component), then because of the high density in the shell in the first hours they will largely be destroyed, and their energy will be dissipated in the form of neutrinos, $\gamma$-rays, and electrons and positrons which radiate in the magnetic field. A large flux of high energy $(\geqslant 100 \mathrm{MeV}) \gamma$-rays will thus be generated and we might expect fluxes to escape over this period at a rate of perhaps $10^{44}-10^{45} \mathrm{erg} / \mathrm{s}$. For reasonnable magnetic field values the synchrotron radiation will not lie in the $\mathrm{X}$-ray or $\gamma$-ray range. However, it is possible that some part of the electron-positron flux will be dissipated by Compton collisions with thermal photons in which $\gamma$-rays are emitted. It is obvious that these suggestions are highly speculative. However, it is clear that detection of a supernova explosion by $\mathrm{X}$-ray and $\gamma$-ray telescopes would give much information on the conditions at the early phases. For example, if there are no high energy $\gamma$-rays emitted this might be interpreted as meaning that there was no early generation of a large flux of relativistic protons.

We turn now to a consideration of the hard radiation from the Crab some 1000 years after 
the supernova exploded. This question was considered by SAVEDOFF (1959). The Crab is one of the strongest radio sources in the sky and both its radio and optical (polarized) continuum are undoubtedly due to synchrotron radiation by relativistic electrons which are capable of producing energetic quanta by a number of processes. In this section we shall consider what can be inferred about the nature of the physical processes in the Crab from observations of the photon spectrum at high energies. Our discussion of possible neutrino emission from the Crab will be given later in Section VI.

Observations of continuum radiation emitted by the Crab have been made in essentially three frequency ranges : the radio range, the optical range (see O'DELL 1962), and the X-ray range (BowYRR et al. 1962) ; Figure 5 summarizes the results. The radio spectral flux $\mathrm{F}_{\mathrm{v}}\left[\mathrm{w} \cdot \mathrm{m}^{-2} \mathrm{~Hz}^{-1}\right]$ is of the form $\mathrm{C}_{r} v^{\alpha}$, where $\alpha=0.27$, and $\mathrm{C}_{r}$ can be determined by the value

$$
\text { [1.23 } \left.\times 10^{-23} \mathrm{w} \cdot \mathrm{m}^{-2} \mathrm{~Hz}^{-1}\right]
$$

af $F_{v}$ at $v=400 \mathrm{MHz}$ (CoNway, KmLlmRmanN, ond LoNG 1963). The synchrotron spectrum apparently retains this form up to a frequency $v_{m}=10^{14} \mathrm{~Hz}$ at the beginning of the optical region. Designating this region $v<v_{m}$ as the radio range the radio luminosity $L_{r}$ can then be computed from an assumed distance $d=1030 \mathrm{pc}$ to the Crab :

$$
\text { (38) } \begin{aligned}
\mathrm{L}_{r}=4 \pi d^{2} \int_{0}^{v_{m}} \mathrm{C}_{r} \nu^{-\alpha} d \nu & \\
=4 \pi d^{2} \mathrm{C}_{r}(1-\alpha)^{-1} & \nu_{m}^{1-\alpha} \\
& \approx 7.4 \times 10^{36} \mathrm{erg} / \mathrm{s} .
\end{aligned}
$$

The luminosity in, for example, the visible range $\left(\nu=4-8 \times 10^{14} \mathrm{~Hz}\right)$ of the optical region is

$$
\mathrm{L}_{\text {。 }} \approx 1.7 \times 10^{36} \mathrm{erg} / \mathrm{s},
$$

while the luminosity in the region $(\lambda=1.5-8 \mathrm{~A})$ of the X-ray observations is also

$$
\mathrm{L}_{\infty} \approx 1.7 \times 10^{36} \mathrm{erg} / \mathrm{s} .
$$

Assuming the radio spectrum $\mathrm{C}_{r} v^{\alpha}$ is due to synchrotron emission by relativistic electrons, an energy spectrum

$n_{e}\left(\gamma_{e}\right)=\mathrm{K}_{e} \quad \gamma_{e}^{-\Gamma_{e}} \quad$ with $\quad \Gamma_{e}=1+2 \alpha=1.54$ is implied. If the mean magnetic field in the Crab is $\mathrm{H}=10^{-4}$ gauss (O'DeLl 1962) the Larmor frequency is $v_{\mathrm{L}}=280 \mathrm{~Hz}$, and the frequency $v_{m}=10^{14} \mathrm{~Hz}$ would be emitted primarily by electrons with

$$
\left(\gamma_{e}\right)_{m}=\left(v_{m} / \nu_{\mathrm{L}}\right)^{1 / 2} \approx 6.0 \times 10^{5} .
$$

The optical radiation from the Crab would be emitted by slightly higher energy electrons. I9 the radio emission originates from a volume $\int d \mathrm{~V}=\mathrm{V}_{0}$, the radio flux $\mathrm{F}_{\mathrm{v}}$ is related to $\mathrm{V}_{0}, d$, $\mathrm{K}_{e}, \mathrm{H}$ by [see Eq. (34)].

$$
\mathrm{F}_{v} \approx\left(12 \pi d^{2}\right)^{-1} \mathrm{~V}_{0} \mathrm{~K}_{\mathrm{e}} c r_{0}^{2} \mathrm{H}^{2} \nu_{\mathrm{L}}^{(\Gamma /-8) / 2} \nu^{(\Gamma-1) / 2} \text {. }
$$

From the value of the product $V_{0} K_{c}$ determined from the radio brightness we can compute the total energy of the radio electrons in the Crab.

$$
\text { (40) } \begin{aligned}
& \mathrm{E}_{r}=\int d \mathrm{VK}_{e} m_{e} c^{2} \int d \gamma_{e} \gamma_{e}^{\left.-\Gamma_{e-1}\right)} \\
= & \mathrm{V}_{0} \mathrm{~K}_{e} m_{e} c^{2}\left(2-\Gamma_{e}\right)^{-1}\left(\gamma_{e}\right)_{m}^{2-\Gamma_{e}} \\
\approx & 1.0 \times 10^{48} \mathrm{erg} .
\end{aligned}
$$

The age $\tau$ of the Crab is 910 years and we see that $\mathrm{E}_{r} / \tau=3.5 \times 10^{37} \mathrm{erg} / \mathrm{s} \gg \mathrm{L}_{r}, \mathrm{~L}_{r}, \mathrm{~L}_{x}$.

For the assumed magnetic field $10^{-4}$ gauss the electrons lose energy at a rate

[E]. (13)] $-\gamma_{e}^{-1}\left(d \gamma_{e} / d t\right) \approx \gamma_{e} \times 1.94 \times 10^{-17} s^{-1}$; for $\gamma_{e} \leqslant 1.8 \times 10^{6}\left(\nu=\nu_{\mathrm{L}} \gamma_{e}^{2} \leqslant 9.0 \times 10^{14} \mathrm{~Hz}\right)$, $-\gamma_{e}^{-1}\left(d \gamma_{e} / d t\right)<\tau^{-1}$.

Thus, for the radio and optical electrons the characteristic time for energy loss by synchrotron emission is greater than the age of the nebula. The rough coincidence of the critical electron energy and synchrotron emission frequency with the value (Fig. 5) above which the spectrum is apparently reduced or perhaps cut off may be interpreted as an indication that the relativistic electrons in the Crab were produced in the initial

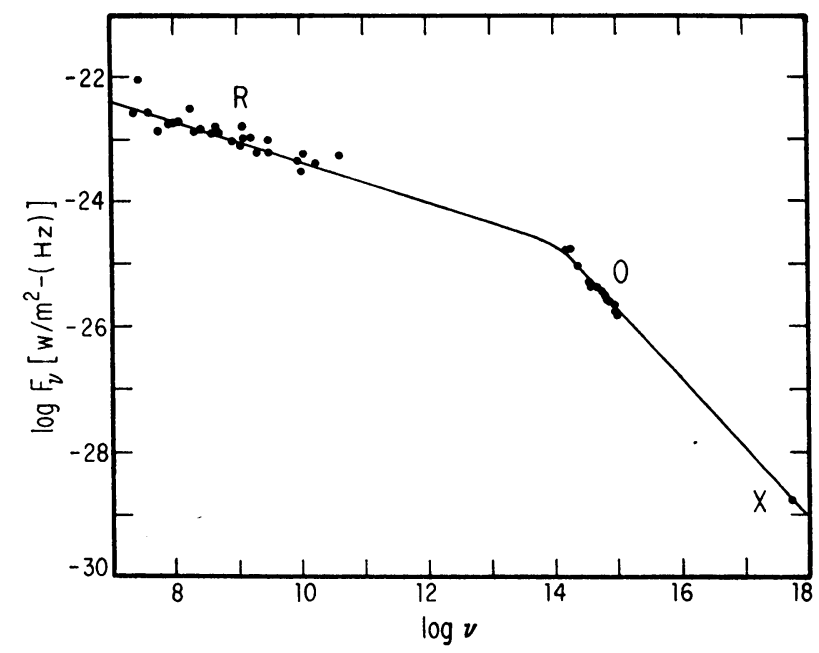

Fig. 5. - Observed radiation from the Crab Nebula in the radio ( $\mathrm{R})$, optical $(\mathrm{O})$ and $X$-ray $(\mathrm{X})$ regions. 
supernova outburst. The absence of any continuous production of high energy electrons would preclude any interpretation of the X-ray point in Figure 5 as being due to electron synchrotron emission, since the lifetime against energy loss through synchrotron emission by the energetic electrons necessary to produce this synchrotron frequency is about 35 years $\ll \tau$. An important parameter in this discussion is the strength of the magnetic field in which the electrons radiate. We have chosen a value of $H=10^{-4}$ gauss so that the lifetimes of the radio and optical electrons are longer than $10^{3}$ years. However, if the assumed value of $H$ is increased perhaps to $5 \times 10^{-4}$ gauss, the lifetimes of the electrons emitting the same synchrotron frequencies are decreased by a factor of $(5)^{3 / 2}(=11.2)$ and the optical electrons have lifetimes less than the age of the nebula so that continuous injection of such electrons is required to explain the optical radiation. Since the magnetic field strength is uncertain we shall consider the possibility of continuous injection of electrons in what follows. We also might mention that as Ginzbura, Pikelner, and SHKLovsky (1955) have shown, there might exist in the Crab an energy loss by scattering by magnetic field condensations in the expanding nebula. These scatterings lead to a Fermi-type statistical deceleration of the electrons. The corresponding energy loss is approximately $-d \gamma_{e} / d t \approx \gamma_{e} \mathrm{~V} / r$, where $\mathrm{V}$ is the expansion velocity of the nebula and $r$ its size; thus $r / V \approx \tau$, the age of the nebula. This energy loss process, if it is operative, dominates synchrotron losses for the radio and optical electrons but is negligible for higher energy electrons. With only this type of energy loss $\left(\propto \gamma_{e}\right)$ the electron spectrum $n_{e}\left(\gamma_{e}\right)$ retains the power law shape of its production spectrum $q_{e}\left(\gamma_{e}\right)$.

Consider the case where the radio electrons of the Crab are produced continuously and, for simplicity, at a constant rate since the origin of the nebula. Neglecting energy losses $\left({ }^{1}\right)$ the continuity equation (24) reduces to

$$
\partial n_{e}\left(\gamma_{e}\right) / \mathrm{\iota} t=q_{e}\left(\gamma_{e}\right)=k_{e} \gamma_{e}^{-\Gamma_{e}},
$$

and so the electron spectrum at the present times would have become

$$
n_{e}\left(\gamma_{e}\right)=\tau q_{e}\left(\gamma_{e}\right)=\mathrm{K}_{e} \gamma_{e}^{-\Gamma_{e}} .
$$

If the continuous production is via meson production in nuclear collisions, as was proposed by

(1) A similar result would be obtained if the Fermi-type statistical deceleration were operative since the characteristics loss time for this process is approximately $\tau$, the age of the nebula. one of us (BURBIDGE 1958), there will also be continuous production of $\pi^{0}$-decay photons, and it is of interest to compute the resulting $\pi^{0}$-photon flux. For a pion production spectrum

$$
q_{\pi}\left(\gamma_{\pi}\right)=k_{\pi} \gamma_{\pi}-\Gamma_{\pi}
$$

the $\pi^{0}$-decay photon production spectrum is approximately

$$
d n \mathbf{0} / d \eta d t \approx{ }_{3}^{2} k_{\pi}\left(2 m_{e} / m_{\pi}\right)-\left(\Gamma_{-}-1\right) \eta-\Gamma_{\pi}
$$

in which it is assumed that $\frac{1}{3}$ of the pions produced are $\pi^{0} s$ and each $\pi^{0}$ of energy $\gamma_{\pi} m_{\pi} c^{2}$ decays into two photons of half this energy. The observed spectral flux of $\pi^{0}$-photons would then be

$$
j^{0}(\eta)=d J / d \eta=\left(4 \pi d^{2}\right)^{-1} \int d v\left(d n^{0} / d \eta d t\right) .
$$

Employing the relation (8) between $k_{\pi}$ and $k_{e}$ and equations (39) and (41) to determine $k_{\pi}$ from the radio spectrum we find

$$
j^{0}(\eta)=1.0 \times 10^{-4} \times \eta^{-1.54}
$$

For photons of energy around

$$
\eta=200(\mathrm{E} \sim 100 \mathrm{MeV})
$$

the integrated spectrum with $\Delta \eta / \eta \sim 1$ gives $\int j^{0}(\eta) d \eta \sim 10^{-4} \eta^{-0.54} \sim 5.7 \times 10^{-6}$ photons $/ \mathrm{cm}^{2}$-s. This photon flux is almost four orders of magnitude smaller than the upper limit established by KraUshaAR and Clark.

One can also calculate the high energy proton flux required to produce the pion production rate necessary to account for the secondary electron density and the radio spectrum. From this proton flux one can then compute the amount of $\mathrm{K}$-series and $\mathrm{X}$-rays in the wavelength range of the observations of Bowyen et al. produced following $K$-shell ionization. The calculated $\mathrm{X}$-ray flux, for a low energy proton cut-off $\gamma_{p}=1$ is about 7 orders of magnitude smaller than the observed flux.

A more definite conclusion regarding secondary electron production in the Crab Nebula may be provided by an analysis of the observations of FroIN et al. (1964). By employing Cerenkov light detectors to observe light pulses from showers in the atmosphere they were able to set upper limits for the high energy photon flux from the Crab Nebula and also from the quasi-stellar radio sources 3C 147, 3C 196 and 3C 273. The threshold energy for their detection system was $5 \times 10^{12} \mathrm{eV}$ 
$\left(\eta \approx 10^{7}\right)$. The established upper limits of the photon fluxes are listed in Table IV.

TABLE IV

UPPER LIMITS TO THE HIGH ENERGY PHOTON FLUX FROM VARIOUS SOURCES (After Fruin et al.)

Source
Crab Nebula
3C 147
3C 196
3C 273

Photon Fudx

(photons $/ \mathrm{cm}^{2}$-s)

$$
\begin{aligned}
& 1 \times \overline{10-10} \\
& 1 \times 10^{-10} \\
& 5 \times 10^{-11} \\
& 3 \times 10^{-10}
\end{aligned}
$$

If photons of energy $\eta=10^{7}$ result from the decay of $\pi^{0^{\prime}} s$ produced in nuclear collisions, the corresponding synchrotron emission frequency in the Crab's magnetic field by electrons resulting from the decay of charged pions of the same energy is about $\nu=10^{16} \mathrm{~Hz}$ for $\mathrm{H}=10^{-4}$ gauss. This frequency is about midway (on the logarithmic scale) between the optical and X-ray frequencies at which the Crab has been observed (see Fig. 5). It is of interest to compute the $\pi^{0}$-photon flux at $\eta=10^{7}$ from the Crab on the assumption that the optical - X-ray flux (if it exists) from the Crab is due to synchrotron emission by secondary - produced electrons.

In the region around $\nu=10^{16} \mathrm{~Hz}$; the apparent index of the synchrotron spectrum (Fig. 5) $\alpha=1.1$, so that the electron spectrum in this region is of the form

$$
n_{e}\left(\gamma_{e}\right)=\mathrm{K}_{e} \gamma_{e}^{-\mathrm{T} e} \text { with } \Gamma_{\epsilon}=3.2 .
$$

Moreover, for these high energy electrons the dominant energy loss process is synchrotron emission and $K_{e}$ is related to the electron production spectrum

$$
q_{e}\left(\gamma_{e}\right)=k_{e} \gamma_{e}^{-\mathrm{T} \pi}\left(\Gamma^{\pi}=\Gamma_{e}-1\right)
$$

by [cf. Section $\operatorname{III}(e)$, Eq. (28)]

$$
\mathrm{K}_{e}=k_{e} / b\left(\Gamma_{\pi}-1\right) \text {, }
$$

with $k_{e}$ related to $k_{\pi}$ by equation (8). Calculating the $\pi^{0}$-photon flux as in equations (41) and (42) and again determining the parameter

$$
\left(4 \pi d^{2}\right)^{-1} V^{0} k_{\pi}
$$

from the supposed synchrotron emission rate

$$
\left[\mathrm{F}_{\nu} \approx 1.4_{5} \times 10^{-27} \mathrm{w} / \mathrm{m}^{2} \mathrm{~Hz} \text { at } \nu=10^{16} \mathrm{~Hz}\right]
$$

one calculates a $\pi^{0}$-photon spectrum given by

$$
j^{0}(\eta)=2.2_{1} \eta^{-2.2} \text {. }
$$

For photons of energy $\eta=10^{7}$ we find the integrated spectrum with $\Delta \eta / \eta \sim 1$ gives

$\int^{\circ} j^{0}(\eta) d \eta \sim 2.2_{1} \eta^{-1.2} \sim 8 \times 10^{9}$ photons $/ \mathrm{cm}^{2} . \mathrm{s}$.

This calculated photon flux is almost two orders of magnitude above the observational upper limit (Table IV). Thus, the present preliminary observations are inconsistent with the interpretation of the X-ray emission from the Crab as synchrotron radiation if the necessary continuous production of high energy electrons is through secondary production via $\pi-\mu-e$ decay. If electrons are produced by secondary processes at a lower energy and then accelerated by Fermi processes to energies at which they will radiate synchrotron $\mathrm{X}$-rays, it may be possible to explain the observed X-ray flux vithout coming into conflict with the results of FruIN et al.

In summary, regarding synchrotron radiation and the relativistic electrons in the Crab, provided that the magnetic field is as weak as $10^{-4}$ gauss, the view that the energetic electrons responsible for the radio and optical radiation in the Crab were produced in the initial supernova outburst is quite consistent. In fact, the apparent reduction below the extrapolated radio spectrum $\mathrm{F}_{\nu}=\mathrm{C}_{r} \nu^{-\alpha}$ in the optical region may possibly be interpretated as a result of energy losses by the more energetic electrons ; that is, higher energy electrons would have already decayed in energy since the birth of the nebula. On the other hand, the electrons required to produce synchrotron radiation on the $\mathrm{X}$-ray region would have to be continuously produced.

It should be pointed out that $\mathrm{X}$-rays can also be emitted by the synchrotron process if electrons spiral into regions of much higher magnetic field. Since the critical frequency is proportional to $H_{\perp}$ this means that the field must be increased by a factor of $\left(v_{x} / v_{0}\right) \sim 10^{3}$. Thus this would imply that there are regions in the Crab with magnetic field strengths as high as $10^{-1}$ gauss. There are many difficulties associated with such a model partly because it would require continuous production of particles which move into regions of high field, since the lifetimes are proportional to $\left(\mathrm{H}_{\perp}\right)^{-2}$. Also, the mechanism by which such concentrations of magnetic flux can be maintained is difficult to understand. These questions will be considered in more detail in a separate paper. 
Finally, we should like to mention another possible explanation for the observed X-rays from the Crab, namely the recent suggestion by HeIrES (1964). According to HeIres the high temperature region behind the shock front of the nebula expanding into the interstellar gas would emit thermal bremsstrahlung $\mathrm{X}$-rays at a rate corresponding roughly to that observed for the Scorpius and Crab Nebula sources. This problem probably needs further investigation ; among other things, it is not clear how the required shock wave is initially produced.

Apart from the generation of hard radiation by the processes described above in the supernova explosion which gave rise to the Crab, considerable amounts of radioactive nuclei may have been synthesized (BURBIDGE, BURBIDGE, FowLER and HoYLe 1957). While in more recent years it has been proposed that the light emitted in a supernova outburst may not be predominantly due to such elements as $\mathrm{Cf}^{254}$, (cf. Hoyle, FowLER, BURBIDGE and BURBIDGE 1964) a test of the different hypotheses may be possible. The flux of $\gamma$-rays emitted by such nuclei as $\mathrm{Cf}^{251}, \mathrm{Cf}^{249}$, $\mathrm{Am}^{243}, \mathrm{Am}^{241}, \mathrm{U}^{233}, \mathrm{~Pa}^{231}$ and $\mathrm{Ra}^{226}$ has been estimated by SavedofF (1959). The energies lie in the $100 \mathrm{KeV}$ range and the calculated fluxes range from $0.011 \mathrm{~cm}^{-2} \mathrm{~s}^{-1}$ (from $\mathrm{Cf}^{251}$ ) to $4.2 \times 10^{-5}$ (from $\mathrm{U}^{233}$ ).

\section{Extragalactic discrete sources.}

The only discrete sources of high energy cosmic photons which have been established with some certainty are the Scorpius source from which an energy flux of $J_{\mathrm{E}} \sim 10^{-7} \mathrm{erg} / \mathrm{cm}^{2}$-s is observed in the $\mathbf{X}$-ray region and the Crab Nebula from which a flux about 1/10 as large is observed. It is of interest to consider the possibility that the Scorpius source is of extragalactic origin as well as to consider the common assumption that it is a galactic object. If the Scorpius source were at a typical galactic distance (that is, within our own Galaxy) $d_{0} \sim 10 \mathrm{kpc}$, its X-ray luminosity would be

$$
\mathrm{L}_{0}=4 \pi d_{0}^{2} \mathrm{~J}_{\mathrm{E}} \approx 10^{39} \mathrm{erg} / \mathrm{s} .
$$

If it were at a typical inter-galactic distance (the distance to a nearby galaxy) $d_{i-o} \sim 1 \mathrm{Mpc}$, its luminosity would be $\mathrm{L}_{i-g} \sim 10^{43} \mathrm{erg} / \mathrm{s}$, while if it were at a cosmological distance (to a distant galaxy) $d_{c} \sim 1000 \mathrm{Mpc}$, its luminosity would be $\mathrm{I}_{c} \sim 10^{49} \mathrm{erg} / \mathrm{s}$. We now make several obervations concerning the energetics of the pro- blem of establishing the distance to and nature of the Scorpius source. For a supernova remnant of age

$$
\tau_{s} \sim 10^{10} \mathrm{~s}, \quad \mathrm{~L}_{g} \tau_{s} \sim 10^{49} \mathrm{erg} .
$$

Since this is comparable with the light energy emitted by a supernova and also with the kinetic energy, and may be some fraction of the total energy released, this means that a galactic supernova at a distance $\sim 10 \mathrm{kpc}$ could supply the energy radiated in $\mathrm{X}$-rays by the Scorpius source for only a relatively short time $\left(\sim 10^{10} \mathrm{~s}\right)$. On a cosmic time scale $\left(^{1}\right) i_{c} \sim 10^{10} \mathrm{yr}$. the energy $\mathrm{L}_{i-o} \tau \sim 3 \times 10^{60}$ ergs is small compared with the optical energy radiated by a normal galaxy ( $\sim 10^{62} \mathrm{erg}$ ), but a normal galaxy would be expected to radiate a very much smaller amount of energy in X-rays. No unusual external galaxies are observed in the direction of the Scorpius source which is about $20^{\circ}$ off the galactic center, although interstellar extinction of our own Galaxy prevents observations at lower galactic latitudes (say $\leqslant 10^{\circ}$ ). However, there are no strong radio sources in the direction of Scorpius. Regarding the possibility that the Scorpius source is a distant galaxy, we note that $\mathrm{L}_{c} \tau_{c} \sim 3 \times 10^{66} \mathrm{erg}$, much greater even than the rest mass energy $\mathbf{M}_{o} c^{2}$ of a galaxy. Moreover, in the matteranti-matter annihilation of a galactic mass which we might conceive took place in a time $\ll \tau_{c}$, the photon energies would be $\geqslant 0.5 \mathrm{MeV}$, not $\mathrm{X}$-ray $(\mathrm{keV})$ energies. On the other hand, the size of a small radio source, for exemple a quasistellar object, is $s \sim 10 \mathrm{kpc}$ and the time $\tau_{s}$ for a light signal to propagate this distance is $s / c \sim 10^{12} \mathrm{~s}$. The product $\mathrm{L}_{c} \tau_{s}$ is then $\sim 10^{61} \mathrm{erg}$ roughly the energy $\mathrm{E}_{r}$ of strong radio sources which may be stored in the relativistic particles.

In summary, it appears that normal distant galaxies (including radio galaxies) are incapable $\left({ }^{2}\right)$ of producing the observed energy flux $\mathrm{J}_{\mathrm{E}}$ corresponding to the Scorpius source over evolutionary time scales $\sim 10^{10} \mathrm{yr}$. However, an outburst over a shorter time might be capable energetically of producing the required X-ray luminosity. Let us consider further such a hypothetical outburst in a galaxy at a distance $d$ involving the release of an amount $\mathrm{E}$ of energy, of which a

(1) This time is also roughly the characteristic time for the evolution of a galaxy.

(2) For example, we compute an X-ray flux from the CoMpton process in the quasi-stellar radio source $3 \mathrm{C} 48$ about 9 orders of magnitude smaller than the flux from the Scorpius source. The source $3 \mathrm{C} 48$ is a small radio object with a high optical luminosity, so that the thermal photon density in the region of the radio electrons is fairly high. 
fraction $f_{\gamma}$ is emitted in high energy photons of mean energy $\overline{\mathrm{E}}_{\gamma}$. If the outburst occurs during a time $\tau$, the observed resulting photon flux would be

$$
J_{\gamma}=\frac{f_{\gamma} \mathbf{E} / \overline{\mathbf{E}}_{\gamma}}{4 \pi d^{2} \tau}
$$

For $\mathrm{E}=10^{60} \mathrm{erg}, d=1000 \mathrm{Mpc}$ and with $\overline{\mathbf{E}}_{\gamma}$ in $\mathrm{MeV}$ and $\tau$ in years we have

$$
J_{\gamma} \approx 170 f_{\gamma} / \overline{\mathrm{E}}_{\gamma} \tau \text { photons } / \mathrm{cm}^{2}-\mathrm{s},
$$

and for $\overline{\mathbf{E}}_{\gamma} \sim 100 \mathrm{MeV}$ (mean energy from $\pi^{0}$-decay) and $\tau \sim 1000 \mathrm{gr}$ (time scale for outburst), $\mathrm{J}_{\gamma} \sim 10^{-3} f_{\gamma}$ photons $/ \mathrm{cm}^{2}$-s. Unless $f_{\gamma}$ is very small, a flux of this magnitude could be observable. The detection of such a discrete source of $\gamma$-rays (or X-rays) might then possibly be interpreted as the observation of the birth of a strong radio source. Finally, we might mention that DuthIE et al. report a possible $(\sim 100 \mathrm{MeV})$ $\gamma$-ray flux of $\sim 0.002$ photons $/ \mathrm{cm}^{2}-\mathrm{s}$ from Cygnus A which is at a distance $\sim 100 \mathrm{Mpc}$.

\section{NeUTRINO SOURCES.}

Any review of the fluxes of hard radiation which may be present in the universe would not be complete without mention of neutrinos. In principle detection of neutrino fluxes would give valuable direct evidence concerning conditions in stellar interiors and also if high energy neutrinos could be observed information on the high energy particle flux could be obtained. Moreover, evidence of the energy density of neutrinos in the universe may have cosmological significance. The subject of neutrino astronomy has been discussed and reviewed ad nauseam in the last two or three years following developments in the theory of weak interactions and the realization that neutrino emission processes will become the dominant energy loss mechanism in the final stages of stellar evolution. Recent papers and reviews which have given some account of these processes and their repercussions on stellar evolution, nucleosynthesis, supernovae and cosmology and in which full references can be obtained are by Pontecorvo (1963), Fowler and HoYle (1964), Burbidat (1962), Whinbera (1962), Fodor. Körvissy and MarX (1964) and Chro (1964). We only give a very brief summary here.

While the energy density in the flux of neutrinos is very considerable, so that, for example, for a normal galaxy it will be some $4 \%$ of the total luminous flux or about $4 \times 10^{42} \mathrm{erg} / \mathrm{s}$, the very small interaction cross-sections $\left(\sim 10^{-44} \eta_{v}^{2} \mathrm{~cm}^{2}\right)$, (unless resonances are present, cf. below) obviously make the fluxes very difficult of detection. Moreover no method of detecting low energy neutrinos with energies below those necessary to induce inverse beta decays is known. We illustrate the problems by discussing the work on solar neutrinos and then consider fluxes from more distant stars and galaxies.

Neutrinos $\left(v_{e}\right)$ are emitted in the normal hydrogen burning processes in stars. About $2 \%$ of the energy released in the p-p chaih and about $6 \%$ in the CNO cycle is emitted as neutrinos. At present there is no feasible way known of detecting neutrinos emitted in $\mathrm{H}^{1}(p, e v) \mathrm{D}^{2}$ because they have a maximum energy of $0.42 \mathrm{Mev}$ and are thus not able to induce most inverse beta decays.

Undoubtedly the Sun is likely to be the strongest apparent source of neutrinos and direct detection of them is of the greatest importance. Following an early suggestion of PoNThCORvo, BAHCALI (1964) and DavIs (1964) have considered in detail the possibility of the detection of neutrinos emitted in $\mathrm{Be}^{7}(e-v) \mathrm{Li}^{7}$ and $\mathrm{B}^{8}\left(e^{+}, v\right) \mathrm{Be}^{8}$ through their absorption $\mathrm{Cl}^{37}\left(v, e^{-}\right) \mathrm{Ar}^{37}$; the activity of $\mathrm{Ar}^{37}$ is then measured. On the basis of the best solar models (Sears 1964) Bahoall has estimated that the fluxes at the earth's surface will be $1.2 \times 10^{10}$ neutrinos $/ \mathrm{cm}^{2} / \mathrm{s}$ and $2.5 \times 10^{7} / \mathrm{cm}^{2} \mathrm{~s}$ from the decay of $\mathrm{Be}^{7}$ and $\mathrm{B}^{8}$ respectively. From BaHCALL's analysis of the cross-sections for $\mathrm{Cl}^{37}\left(v, e^{-}\right) \mathrm{Ar}^{37}$ Davis has concluded that the expected neutrino captures in $10^{5}$ gallons of $\mathrm{C}_{2} \mathrm{Cl}_{4}$ in a mine would be about 4- 11 a day which would be an order of magnitude above the background produced by the production of $\mathrm{Ar}^{37}$ by cosmic rays underground through $\mathrm{Cl}^{37}(p, n) \mathrm{Ar}^{37}$. The flux of detectable neutrinos from the central bulge of the galaxy will be less than that from the Sun by a factor $10^{7}-10^{8}$ while the flux to be expected from a nearby galaxy such as M31 would be less than the Sun by a factor $\sim 10^{11}$. While neutrinos are emitted in the normal energy producing cycles in the stars, neutrinos and anti-neutronos are emitted with positrons and electrons respectively by beta unstable nuclei in the processes of energy generation and element synthesis beyond hydrogen. However, for a galaxy in a steady state it is easily shown that the fluxes to be expected are small compared with those emitted in hydrogen burning.

In the high temperature phases of stellar evolu- 
tion (for core temperatures $\geqslant 5 \times 10^{8}$ degrees) neutrino pair emission becomes the dominant mechanism of energy loss. They arise by a variety of reactions in all of which they replace photon emission. An important process is

$$
e^{+}+e^{-} \rightarrow v+\bar{v} \text {. }
$$

While this mechanism of energy loss is important from the point of view of the evolutionary process, an individual object (perhaps the immediate forewarning of a supernova) would be very difficult to detect even if a mechanism of detecting low energy neutrinos were found, because of the very short time scale associated with such evolutionary phases. Thus, for exemple, FowLER and HOYLE (1964) have calculated that if one solar mass in the center of a massive star reaches a temperature of $3.5 \times 10^{9}$ degrees, the neutrino flux will amount to $\sim 10^{47} \mathrm{erg} / \mathrm{s}$. However, this phase will only last a few seconds. At a later stage, after a star has exploded and if a neutron configuration remains, the initial neutrino flux for a core temperature of $2 \times 10^{9}$ degrees will be about $4 \times 10^{39} \mathrm{erg} / \mathrm{s}$ (CHIU and SALPETER 1964) and the time scale associated with this phase will be about 10 years.

We turn finally from the low energy neutrinos emitted in stellar evolution to consider the possibility as to whether high energy neutrinos $\left(E_{v} \geqslant 100 \mathrm{MeV}\right)$ are emitted in supernova outbursts and from radio sources in which large fluxes of high energy particles are present. Neutrinos are produced whenever a flux of high energy nuclides (protons) interacts with the nuclei of the local gas atoms to produce pions. In the $\pi \rightarrow \mu \rightarrow e$ decay of the charged pions both neutrinos and antineutrinos of the electron and muon type result. That is, in the pion decay

$$
\begin{aligned}
& \pi^{+} \rightarrow \mu^{+}+{ }^{\prime}, \\
& \pi^{-} \rightarrow \mu^{-}+\mu,
\end{aligned}
$$

while in the muon decay

$$
\begin{aligned}
\mu^{+} & \rightarrow e^{+}+\nu_{e}+\bar{\nu}_{\mu}, \\
\mu^{-} & \rightarrow e^{+}+\bar{\nu}_{e}+\nu_{\mu} .
\end{aligned}
$$

Thus, a single charged pion pair $\pi^{+}, \pi$ results in

$$
2\left(\nu_{\mu}+\bar{\nu}_{\mu}\right)+\left(\nu_{e}+\bar{\nu}_{e}\right) ;
$$

twice as many $\mu$-neutrinos as $e$-neutrinos are produced. In the pion decay the muon is essentially non-relativistic in the rest frame of the pion and most of the energy is carried away by the neutrino; here

$$
\mathrm{E}_{\nu} \approx\left(m_{\pi}-m_{\mu}\right) c^{2} \approx \frac{1}{4} m_{\pi} c^{2} .
$$

In the muon decay the mean neutrino energy is about

$$
\frac{1}{3} m_{\mu} c^{2} \approx \frac{1}{4} m_{\pi} c^{2}
$$

in the rest frame of the muon and pion (see Sect. II $b$ ). Thus, the mean lab energy of the neutrinos in both decays is about

$$
\frac{1}{4} \gamma_{\pi} m_{\pi} c^{2}=\frac{1}{4} \mathrm{E}_{\pi} \text {. }
$$

The (anti) neutrino production spectrum is readily computed from the pion production spectrum and is of the form similar to that for the production spectrum of $\pi^{0}$-decay photons [see Eq. (41)], that is, $d n_{v} / d \eta_{v} d t \propto \eta_{v}^{-\Gamma_{\pi}}$, where $\Gamma_{\pi}$ is the index of the pion production spectrum. The ratio of the (anti) neutrino production spectrum (or of the spectral flux) to the $\pi^{0}$-decay photon spectrum at the same $\eta$ is, assuming equal numbers of $\pi^{+}, \pi, \pi^{0}$ produced, roughly

$$
\begin{aligned}
& \left(\nu_{\mu} / \gamma\right)_{\text {same }} \eta \approx 2-\left(\Gamma_{\pi-2)}\right. \\
& \left(\nu_{e} / \gamma\right)_{\text {same }} \eta \approx 2^{-(\Gamma \pi-1)} .
\end{aligned}
$$

BaHCanl and Frautschi (1964) have discussed the detection of high energy neutrinos and have considered the possibility of observing a neutrino flux from the Crab Nebula and other radio sources. They assume neutrino production via $\pi-\mu$ decay, which implies also continuous production of pions and $\pi^{0}$-decay photons. Assuming a continuous constant production of high energy radio electrons through $\pi-\mu$ decay in the Crab since its birth, the associated $\pi^{0}$-decay photon flux was calculated in Section IV [Eq. (44)]. The corresponding neutrino flux is of the same form

$$
j_{v}\left(\eta_{v}\right)=k \eta_{v}-\Gamma \pi,
$$

with $\Gamma_{\pi}=1.54$. For $\mu$-neutrinos

$$
k_{\mu} \approx 2^{0.46} \times 1.0 \times 10^{-4} \mathrm{~cm}^{-2} \mathrm{~s}^{-1} \text {, }
$$

while for $e$-neutrinos $k_{e} \approx \frac{1}{2} k_{\mu}$. This neutrino spectrum and also the $\pi^{0}$-decay photon spectrum is associated with (if there is continuous production) the radio synchrotron spectrum for $10^{7} \mathrm{~Hz}<\nu<10^{14} \mathrm{~Hz}$ and with electron energies $200<\gamma_{e}<6 \times 10^{5}$. The range of $\eta_{v}$ over which equation (51) should represent the neutrino spectrum is the same as the range of $\gamma_{e}$, that is 
for $100 \mathrm{MeV} \leqslant \mathrm{E}_{\mathrm{v}} \leqslant 300 \mathrm{BeV}$. A neutrino spectrum similar to equation (51) was derived by Bahcall and Fradtschi. However, we doubt that such a neutrino flux will ever be observed from the Crab. In Section IV we showed that there is evidence against continuous production via $\pi-\mu$ decay of very high energy electrons which would produce synchrotron radiation in the optical - X-ray range; moreover, the lifetime against synchrotron losses for the radio and optical electrons in the Crab is longer than the age of the nebula. We therefore feel that probably there is little or no continuous production of radio electrons in the Crab and no associated neutrino or $\pi^{0}$-decay photon production.

Regarding possible neutrino production in other radio sources, in particular in extragalactic objects, similar considerations apply. If there does exist continuous production of radio electrons via $\pi-\mu$ decay, and a steady state exists, then the energy radiated in neutrinos would be comparable to the total energy emitted in synchrotron radiation by the relativistic electrons produced with the neutrinos For " normal" radio galaxies with steep spectra (index $\alpha \approx 0.8$ ) most of the neutrinos produced would have fairly low energies $\left(E_{v} \sim 100 \mathrm{MeV}\right)$, while sources with flat radio spectra (e. g., Crab Nebula, M 82) might be expected to emit predominantly higher energy neutrinos (say, $E_{v} \sim 100 \mathrm{BeV}$ ). The strong extragalactic radio sources and quasistellar objects would be emitting lower energy neutrinos with $\mathrm{E}_{\mathrm{v}} \sim 1 \mathrm{BeV}$ at power levels of $10^{44}-10^{45} \mathrm{erg} / \mathrm{s}$.

However, it appears probable now that such steady state conditions are not present in these sources, so that even if large proton fluxes are present, the neutrino fluxes will be much lower than this (cf. Burbidae, Burbidge and SaNDAGE 1963). On the other hand it is possible that at an early phase when a violent outburst in a galaxy gives rise to some $10^{60} \mathrm{ergs}$ of high energy particles (perhaps over a period of 1000 years), a large fraction of which may be protons, the collisions of some part of these with the interstellar gas before they escape into regions of very low density might give rise to a flux of high energy neutrinos several orders of magnitude greater than the values corresponding to steady state conditions. Thus one might expect to observe both neutrinos and $\pi^{0}$-decay photons from a violent outburst in a galaxy (see Sect. V).

The possibility of detecting such fluxes of high nerg $y$ neutrinos has been considered by BAHCALL and Fradtschi (1964). They have pointed out that the very small cross sections for the interaction of neutrinos with matter mean that from very strong radio sources with a dominant proton flux only one neutrino-induced event per day would be experienced in a $10^{5}$ ton absorber. However, as BaHCaLl and Fradtschi have proposed, the possibility exists that resonances in neutrino interaction processes are present. As they suggest, the reaction

$$
\bar{v}_{e}+e-\rightarrow \bar{v}_{\mu}+\mu^{+}
$$

may have a resonance and may be detected by neutrino interactions with material in the earth's crust. Clearly a great deal of information might be gained from observations of neutrinos from extragalactic objects. Thus the most pressing requirement is to devise a neutrino telescope which has good angular resolution. BABOALL and FraUTSCHI have suggested that the muons ejected in (52) may enable this to be achieved.

\section{Conclusion.}

We have tried to summarize those mechanisms which may give rise to hard radiation in the universe. At present, apart from observations of the Sun, there is little observational evidence which can be used in conjunction with the theoretical estimates. The brilliant work of the NRL and MIT groups has shown that there are sources of $\mathrm{X}$-rays at flux levels which are detectable with present techniques. Moreover the absence of a large isotropic flux of X-rays has enabled us to set limits on the temperature of the intergalactic medium. As far as $\gamma$-rays are concerned it is not yet clear whether high energy $\gamma$-rays are present at the flux levels calculated in Seotion III. The detection of high energy neutrino fluxes would be very exciting but the preliminary results (cf. Cowan et al. 1964) must be viewed with caution.

What are the possibilities for further investigations in this field ? To us the parallel of this field of research with that of the early days in radio astronomy is strong. There is one major difference, however and this concerns the theoretical expectations in the field.

The discovery of significant fluxes of radio emission from the cosmos was totally unexpected, and in the first decade after the war theoreticians only gradually came to understand that the process by which the non-thermal sources radiate is the synchrotron mechanism. Of course the 
process of thermal emission was well understood but could not explain the strength or the spectral characteristics of the bulk of the radiation. During this period there was much confusion because of the unexpected nature of the discoveries and it was the interplay between the theory and optical observation which led to the elucidation of the mechanism by which the sources radiate. The theoretical problem then devolved into that of understanding how the vast fluxes of relativistic particles and magnetic field originate.

As far as the hard radiation is concerned, the physical mechanisms by which such radiation can be emitted are well known and the level at which fluxes have been detected (or not detected) suggests that no objects with the unexpected character of the radio sources are likely to be found by observational techniques in this energy range. If hard radiation is emitted by hot bodies, then they must have very hot surfaces and hence they will cool very rapidly and soon cease to emit hard radiation. Otherwise the mechanisms by which hard quanta are emitted all stem from the interaction of fast charged particles with matter radiation, or magnetic fields. Knowledge gained through cosmic-ray and radio astronomical discoveries enables predictions to be made of the fluxes of hard radiation to be expected with a range of parameters associated with the present uncertainties in these quantities. Thus detection and even non-detection of hard radiation will be most valuable in determining the state of matter and radiation in the universe.

The parallel between the developments in radio astronomy and $X$-ray, $\gamma$-ray, and neutrino astronomy is very close when we consider the problem of the discrete sources. In the early days in radio astronomy resolution was very poor and at least one of the strongest sources was put in the wrong constellation by one notable group of investigators. All of the major developments in the study of discrete radio sources have come in step with the increase in prescision with which positions of sources could be determined. This has enabled the objects to be observed optically with large telescopes. With optical identification has come measurement of distance and with this a beginning of quantitative study of the physical conditions in the sources. It is the absence of a method of determining the distance of an extragalactic source which has required the cooperation of optical and radio telescopes $\left({ }^{1}\right)$. The same situation

(1) In principle the $21 \mathrm{~cm}$ line is a powerful tool for determining distance by redshift measurements, but in practice it appears to apply in $\gamma$-ray and $X$-ray astronomy, since the flux emitted in lines will in general be small. A vast improvement in resolution is required in order to determine better positions for the X-ray sources in the directions of Scorpius and the Crab. The lunar occultation observation of the NRI group is a first step in this direction. Such an improvement in resolution together with greatly increased sensitivity will be required, for example, before it can be established or denied that neutron stars have been detected. In principle the problem is even more difficult than this, since it would be necessary to prove that the object is of stellar size or less, and even if its angular size were shown to be infinitesimally small its distance would still not be known from observation ( $\left.{ }^{1}\right)$. The location of a small source within the Crab would not in itself be adequate proof of the existence of neutron stars since other mechanisms could provide the observed flux (cf. Section IV). Conversely, the establishment of an extended source in the Crab does not definitely rule out the neutron star hypothesis, since a cluster of such stars might be involved, Apart from theoretical arguments concerning the lifetimes of hot neutron stars, determination of the form of the spectrum of the radiation and the setting of limits to the flux of $\gamma$-rays which is present would be a good observational test of the neutron star hypothesis.

It is clear that the various theoretical estimates of fluxes which we have given in this paper suggest that a great increase in sensitivity of detectors as well as good resolution will be needed to exploit this field to the utmost. Finally, it is not out of place to remark that the $X$-ray observations have already shown that the universe is not very hot, and it may in fact be rather cool. In this case, apart from the neutrino flux which is part of the general cosmological thermal radiation field, the flux of hard radiation may be rather weak.

We are indebted to many friends and colleagues who have provided much material prior to publication. This research has been supported in part by the National Science Foundation and in part by NASA through contract NsG-357.

cannot be used since to detect the feature in galaxies at only very modest distances $(<20 \mathrm{Mpc})$ is beyond the capability of present day radio telescopes.

(1) After all, it should be remembered that a persistent heresy in the radio astronomical field is that the large bulk of the sources are nearby stars. There is no direct dispronf of this, though all indirect arguments are against it.

Manuscrit reçu le 19 octobre 1964. 


\section{BIBLIOGRAPHY}

Agrinier B., Kohohim Y., Parlier B., Boklla G., Drght Antoni G., Dilworth C., Scarsi L. and SinonI G., 1964, Phys. Rev. Letters, 13, 377.

Allen C. W., 1963, Astrophysical Quantities (London : Athlone Press).

Alum L. H., 1963, The Atmospheres of the Sun and Stars, 2nd Ed. (New York : Ronald Press).

Arnold J. R., Metzghr A. E., ANDhrion E. C. and VAN DinuA M. A., 1962, J. Geophys. Res., 67, 4878.

BAADE W. and ZWICKY F., 1938, Ap. J., 88, 411.

BaHcall J. N., 1964, Phys. Rev. Letters, 12, 300.

Batoall J. N. and Frautschi S. C., 1964, Phys. Rev., 135, B788.

Bowyme S., Byram E. T., Chubb T. A. and Friedman H., 1964, Nature, 201, 1307.

Burbidge E. M., Burbidge G. R., Fowler W. A. and Hoyst F., 1957, Rev. Mod. Phys., 29, 547.

Burbidge E. M., Burbidge G. R. and Hoyle F., 1963, Ap. J., 188, 873.

Burbidat G. R., 1962, Ann. Rev. Nucl. Sci., 12, 507 ; 1958, Nuovo Cimento Suppl., 8, 403.

Burbidge G. R., Burbidge E. M. and Sandage A. R., 1963, Rev. Mod. Phys., 35, 947.

Burbidan G. R., Gound R. J. and Pottasch S. R., 1963, Ap. J., 138, 945.

Burbidat G. R. and Hoyle F., 1956, Nuovo Cimento, $4,1$.

Burgiss A. and Sfaton M. J., 1964, M. N., 127, 355.

CHI0 H.-Y., 1964, Ann. Phys., 26, 364.

Chro H.-Y. and Salpeter E. E., 1964, Phys. Rev. Letters, 12, 413.

Colatite S., 1963, Proceedings of the Jaipur Conference.

Conway R. G., Kellermann K. J. and Long R. J., 1963, M. N., 125, 261.

Cowan C. L., Ryan D. F. and SzyduIK P. P., 1964, "Experimental Evidence for an Observable Cosmic Neutrino Signal" (preprint).

DatT B., 1938, Zf. f. Ap., 108, 314.

Davis R., Jr., 1964, Phys. Rev. Letters, 12, 303.

Davis R. D., M. N., 128, 133.

DE JAGmR C., 1960, Mém. Soc. R. Sci. Liège, 16, 280.

de Shong J. A. Jr., Hindebrand R. H. and Meyer P., 1964, Phys. Rev. Letters, $12,3$.

Dolan J. F. and Fazio G. G., 1964, " The Gamma-ray Spectrum of the Sun" (preprint).

Donahue T. M., 1951, Phys. Rev., 84, 972.

Duthin J. G., Hafner E. M., KapLoN M. F. and Fazio G. G., 1963, Phys. Rev. Letters, 10, 364.

Elw ERT G., 1952, Z. Naturforsch, 7a, 202, 432 ; 1954, Z. Naturforsch, 9a, 637 ; 1961, J. Geophys. Res., Res., 66, 391.

Ferenberg E. and Primakofr H., 1948, Phys. Rev., $73,449$.

Fruten J. E. and Morrison P., 1963, Phys. Rev. Letters, 10, 453.

FinzI A., 1964a, Ap. J., 139, 774.

Finza A., 1964b, preprint.
Frrkowski R., Garvin J., Maze R. and Zawadzki A., 1962, J. Phys. Soc. Japan, 17, Suppl. A-III, 123.

Fodor L., Kövessy Z. and MARX G., 1964, "Inter-

L. stellar Neutrino Density and Cosmogony" (preprint) to be published in Acta Physica Hungarica.

Fowler W. A. and Horne F., 1960, Ann. Phys., 10, 280 ; 1964, Ap. J. Suppl. 9, 91, 201.

Frmadman H., 1961, Space Astrophysics, ed. Liller. p. 112.

Froin J. H., Jelley J. V., Long C. D., Porter N. A. and Whenks T. C., 1964, Phys. Letters, 10, 176.

Giadooni R., GuRsky H., PaOLINI F. R. and Rossi B. B., 1962, Phys. Rev. Letters, 9, 439.

Ginzbura V. L., Ptk hliner S. B. and Shklovsky J. S., Astr. Zhur., 32, 503.

Ginzburg V. L. and Syrovatsky S. I., 1963, Astr. Zhur., 40, 466 ; 1964, Soviet Phys. J. E. T. P., 18, 245.

Gold T. and HoyLE F., 1958, Paris Symposium on Radio Astronomy, ed. R. N. Bracewell (Stanford : Stanford Univ. Press), p. 583.

Goldreior P. and Morrison P., 1964, Soviet Phys. J. E. T. P., 18, 239.

Gould R. J. and Burbidge G. R., 1963, Ap. J., 138, 969.

Gould R. J., Gold T. and Salphetrir E. E., 1963, Ap. J., 138, 408.

Goumd R. J. and Sclama D. W., 1964, Ap. J., 140, 1634.

Hayakawa S. and Kitao K., 1956, Prog. Theor. Phys., 16, 139.

Hayakawa S. and Matsuoka M., 1963, Prog. Theor. Phys., 29, 612.

Hentes C., 1964, Ap. J., 140, 470.

HeItLer W., 1954, The Quantum Theory of Radiation (London : Oxford Univ. Press).

House L., 1964, Ap. J. Suppl., 8, 307 (No. 81).

Hoyle F., Fowler W. A., Burbidat G. R. and BuRBIDGE E. M., 1964, Ap. J., 139, 909.

JAUCH J. M. and ROHRLICH F., 1955, Theory of Photons and Electrons (Cambridge, Mass : AddisonWesley Publishing Co.).

KIDD J. M., 1963, Nuovo Cimento, 27, 57.

Kradshaar W. L. and Clark G. W., 1962, Phys. Rev. Letters, 8, 106.

LaNDaU L., 1932, Physik, Zeit., Soviet Union, 1, 285 ; 1953, Izv. Akad. Nauk. SSSR, 17, 51.

Marshak R., 1952, Meson Physics (New.York : McGraw-Hill Book Co.).

Misner C. W. and Zapolsky H. S., 1964, Phys. Rev. Letters, 12, 635.

Morrisón P., 1958, Nuovo Cimento, 7, 858.

Morton D. C., 1964, Ap. J., 140, 460.

Nikishev A. J., 1962, Soviet Phys. J. E. T. P., 14, 393.

O'D ful C. R., 1962, Ap. J., 136, 809.

OpPenheimer J. R. and SNYder H., 1939, Phys. Rev., 56, 455. 
Oppenheimer J. R. and Volkoff G. M., 1939, Phys. Rev., 55, 374.

Parker E. N. 1963, Interplanetary Dynamical Processes (New-York : Interscience).

Peterson L., 1964, J. Geophys. Res. (to be published).

Peterson L. E. and Winkler J., 1959, J. Geophy. Res. 64, 697.

Pollack J. B. and Fazio G. G., 1963, Phys. Rev., 131, 2684.

Ponteconvo B., 1963, Soviet Phys. Uspekhi, 6, 1.

Pottason S. R., 1960, $A p$. J., 131, 68.

Savedoff M. P., 1959, Nuovo Cimento, 13, 12.
Solama D. W., 1964a, “ On the Formation of Galaxies and Their Magnetic Fields in a Steady State Universe" (preprint) 1964b, "Cosmic X-Rays as a Tool for Exploring the Large-Scale Properties of the Universe" (preprint).

Sears R. L., 1964, $A p . J ., 140,477$.

Suga K., Escobar J., Clark G., Hazan W., Hendei, A. and Murakami K., 1962, J. Phys. Soc. Japan, 17, Suppl. A-III, 128.

Weinberg S., 1962, Phys. Rev., 128, 1457.

Zwiсky F., 1938, Ap. J., 88, 522.

\section{Discussion}

M. SAVEDOFF. - In early stages of white dwarf formation, we have smaller radii than solar radius, hence possibility of large coronal temperatures. This should be watched as at these stages. the luminosity is quite high, $200 \mathrm{~L}_{\odot}$ and photospheric temperatures are $10^{5} \mathrm{oK}$. Although crude models seem stable against convection, our ignorance of magnetic effects may permit formation of coronae at $\mathrm{T} \sim 5.10^{7} \mathrm{oK}$ with lifetimes of $3.10^{5}$ years.

J. E. Fruten. - It should be pointed out that most strong radio sources will produce gamma rays by inverse Compton effect from the fast electrons which they contain ; in general, however, the expected source strengths due to this process are one or two orders of magnitude below the expected gamma source strengths of the same object due to other processes. It is only in regions of low gas density and magnetic field that the inverse Compton effect becomes important.

G. BURBIDGE. - That is generally true, but in extraordinary objects like quasi-stellar sources.

R. J. GooLD. - Objects, in which the photon density is large.

G. BURBIDGE. - Exactly, in these objects, in which the photon density is very great due to the enormous release of optical energy, the Compton process again contributes significantly.

E. Schatzman. - Dr. Burbidge mentioned the $\gamma$ flash of Supernovae due to nuclear reactions. I think it should be necessary to consider also the gamma-ray emission by synchrotron radiation at the early times of the 1054 Supernova. It is quite possible that for a few days or a few weeks, very energetic electrons could radiate in a magnetic field stronger than the present magnetic field. It is quite difficult to estimate the flux of gamma-rays coming from the Crab nebula at the time of the outburst, as backward extrapolations are quite uncertain. The trouble is that the flux of gamma-rays and X-rays might have been large enough to have a genetic influence on the human beings.

G. BuRBIDGe. - Dr. Gould and I have briefly considered this point in the text which will be published.

A. J. Deutsch. - The Burbidges and Sandage have recently assembled evidence of the occurrence of catastrophic events at the center of a variety of massive galaxies, possibly including our own. On the charts that Dr. FrIEdman has shown the sources in Scorpio and Sagittarius appear to be not too clearly separated from each other. Is it, therefore, possible that both represent the $\mathrm{X}$-rays generated in the remnants of such an event at the center of our Galaxy, X-rays which suffer small-angle scattering in the dust that lies between the center of the Galaxy and the sun?

G. BURBIDGE. - The source at the Galactic center might be due originally to an explosive event. I doubt whether the Scorpius source could be explained in this way. 